\title{
A Bilevel Model for Participation of a Storage System in Energy and Reserve Markets
}

\author{
Nasrolahpour, Ehsan; Kazempour, Jalal; Zareipour, Hamidreza; Rosehart, William D.
}

Published in:

IEEE Transactions on Sustainable Energy

Link to article, DOI:

10.1109/TSTE.2017.2749434

Publication date:

2018

Document Version

Peer reviewed version

Link back to DTU Orbit

Citation (APA):

Nasrolahpour, E., Kazempour, J., Zareipour, H., \& Rosehart, W. D. (2018). A Bilevel Model for Participation of a Storage System in Energy and Reserve Markets. IEEE Transactions on Sustainable Energy, 9(2), 582 - 598. https://doi.org/10.1109/TSTE.2017.2749434

\section{General rights}

Copyright and moral rights for the publications made accessible in the public portal are retained by the authors and/or other copyright owners and it is a condition of accessing publications that users recognise and abide by the legal requirements associated with these rights.

- Users may download and print one copy of any publication from the public portal for the purpose of private study or research.

- You may not further distribute the material or use it for any profit-making activity or commercial gain

- You may freely distribute the URL identifying the publication in the public portal 


\title{
A Bilevel Model for Participation of a Storage System in Energy and Reserve Markets
}

\author{
Ehsan Nasrolahpour, Student Member, IEEE, Jalal Kazempour, Member, IEEE \\ Hamidreza Zareipour, Senior Member, IEEE, and William D. Rosehart, Senior Member, IEEE
}

\begin{abstract}
We develop a decision-making tool based on a bilevel complementarity model for a merchant price-maker energy storage system to determine the most beneficial trading actions in pool-based markets, including day-ahead (as joint energy and reserve markets) and balancing settlements. The uncertainty of net load deviation in real-time is incorporated into the model using a set of scenarios generated from the available forecast in the day-ahead. The objective of this energy storage system is to maximize its expected profit. The day-ahead products of energy storage system include energy as well as reserve commitment (as one of the ancillary services), whereas its balancing product is the energy deployed from the committed reserve. The proposed model captures the interactions of different markets and their impacts on the functioning of the storage system. It also provides an insight for storage system into clearing process of multiple markets and enables such a facility to possibly affect the outcomes of those markets to its own benefit through strategic price and quantity offers. The validity of the proposed approach is evaluated using a numerical study.
\end{abstract}

Index Terms-Energy storage system, energy, reserve, balancing market, strategic bidding

\section{NOMENCLATURE}

\begin{tabular}{|c|c|}
\hline \multicolumn{2}{|c|}{ A. Acronyms used in superscripts } \\
\hline EN & Energy \\
\hline BL & Balancing \\
\hline RS & Reserve \\
\hline UP & Upward reserve \\
\hline DN & Downward reserve \\
\hline $\mathrm{ch}$ & Charging mode of storage system \\
\hline & Discharging mode of storage system \\
\hline \multicolumn{2}{|c|}{ B. Indices and Sets } \\
\hline$t, h$ & Indices of time periods running from 1 to $N_{t}$ \\
\hline$k$ & Index of scenarios running from 1 to $N_{k}$ \\
\hline & Index of generators running from 1 to $N_{g}$ \\
\hline & Index of demands running from 1 to $N_{d}$ \\
\hline & Index of storage systems running from 1 to $N_{s}$ \\
\hline \multicolumn{2}{|r|}{ C. Parameters } \\
\hline$O_{g}$ & Offer price of generator $g,(\$ / M W h)$ \\
\hline$P_{g}$ & Capacity of generator $g,(\mathrm{MW})$ \\
\hline$\Phi_{k}$ & Probability of scenario $k$ \\
\hline$R_{g}$ & Maximum reserve capacity of generator $g$, (MW) \\
\hline$P_{d, t}$ & Maximum load of demand $d$ at time $t,(\mathrm{MW})$ \\
\hline
\end{tabular}

E. Nasrolahpour, H. Zareipour, and W. D. Rosehart are with the Department of Electrical and Computer Engineering, Schulich School of Engineering, University of Calgary, Calgary, AB, Canada T2N 1N4 (e-mail: enasrola@ucalgary.ca; h.zareipour@ucalgary.ca; rosehart@ucalgary.ca).

J. Kazempour is with the Technical University of Denmark, Kgs. Lyngby, Denmark (e-mail: seykaz@elektro.dtu.dk). The work of J. Kazempour is partly supported by the Danish Strategic Council for Strategic Research through the projects of PROAIN (no. 3045-00012B/DSF).
$R_{d} \quad$ Maximum reserve capacity of demand $d$, (MW)

$U_{d, t} \quad$ Bid price of demand $d$ at time $t,(\$ / \mathrm{MWh})$

$V_{d, t} \quad$ Load shedding cost of demand $d$ at time $t,(\$ / \mathrm{MWh})$

$M C_{s}$ Marginal cost of storage system $s,(\$ / \mathrm{MWh})$

$P_{s} \quad$ Charging/discharging capacity of storage system $s$, (MW)

$R_{s}$ Maximum reserve capacity of storage system $s$, (MW)

$E_{s} \quad$ Reservoir capacity of storage system $s$, (MWh)

$E_{s}^{\text {ini }} \quad$ Initial stored energy of storage system $s$, (MWh)

$\alpha_{s} \quad$ A non-negative factor to control stored energy of storage system $s$ for the the next time horizon

$\eta_{s} \quad$ Efficiency of storage system $s$

$R_{t} \quad$ Reserve requirement of the market at time $t$, (MW)

$Q_{t, k} \quad$ Net load deviation at time $t$ under scenario $k$, (MW)

D. Upper-level variables

$e_{s, t} \quad$ Stored energy of storage system $s$ at time $t,(\mathrm{MWh})$

$u_{s, t}$ Binary decision variable indicating the operation mode of storage system $s$ at time $t$

$\widehat{o}_{s, t} \quad$ Price bid/offer by storage system $s$ at time $t,(\$ / \mathrm{MWh})$

$\widehat{p}_{s, t} \quad$ Energy quantity bid/offer by storage system $s$ at time $t,(\mathrm{MW})$

$\widehat{r}_{s, t} \quad$ Reserve capacity bid/offer by storage system $s$ at time $t,(\mathrm{MW})$

E. Lower-level variables (day-ahead market clearing)

$p_{g, t} \quad$ Scheduled production of generator $g$ in day-ahead market at time $t,(\mathrm{MW})$

$r_{g, t}$ Committed reserve from generator $g$ in day-ahead market at time $t,(\mathrm{MW})$

$p_{d, t} \quad$ Consumption of demand $d$ at time $t$, (MW)

$r_{d, t} \quad$ Committed reserve from generator demand $d$ in dayahead market at time $t,(\mathrm{MW})$

$p_{s, t} \quad$ Scheduled energy production/consumption of storage system $s$ in day-ahead market at time $t$, (MW)

$r_{s, t}$ Committed reserve from storage system $s$ in dayahead market at time $t$, (MW)

$\lambda_{t} \quad$ Day-ahead market-clearing price (as a dual variable) at time $t,(\$ / \mathrm{MWh})$.

F. Lower-level variables (balancing market clearing)

$q_{g, t, k}$ Energy deployed from reserve of generator $g$ in balancing market at time $t$ under scenario $k$, (MW)

$q_{d, t, k}$ Energy deployed from reserve of demand $d$ in balancing market at time $t$ under scenario $k$, (MW)

$l_{d, t, k}$ Involuntarily curtailed load of demand $d$ in balancing market at time $t$ under scenario $k$, (MW)

$q_{s, t, k}$ Energy deployed from reserve of storage system $s$ in balancing market at time $t$ under scenario $k$, (MW)

$\lambda_{t, k} \quad$ Balancing market-clearing price (as a dual variable) at time $t$ under scenario $k,(\$ / \mathrm{MWh})$. 
$\mu, \rho \quad$ Dual variables corresponding to inequality day-ahead and balancing lower-level constraints. See Sections (II-B) and (II-C) for details.

\section{INTRODUCTION}

Federal Energy Regulatory Commission (FERC) order 784 allows energy storage facilities to participate in ancillary services market [1]. This has created opportunities for such facilities to expand their revenue streams and improve their economics. Previous studies have also realized that participating in multiple market opportunities improve the economic feasibility of storage projects [2],[3]. Thus, there is a need for better understating the operation strategies of storage systems in energy and ancillary services markets.

In this line, the main purpose of this paper is to determine the optimal bidding and offering strategies of a merchant storage system in multiple pool-based markets, including dayahead market (as a joint energy and reserve settlement), and balancing (real-time) market. The uncertainty of net load (demand minus variable generation) deviation in real-time is incorporated into the model using a set of scenarios generated from the available forecast in the day-ahead. The objective of this energy storage system is to maximize the expected profit. The day-ahead products of energy storage system include energy as well as reserve commitment (as one of the ancillary services), whereas its balancing product is the energy deployed from the committed reserve. The proposed model captures the interactions of energy, reserve, and balancing markets, and their impacts on the functioning of an energy storage system. It also provides an insight for a storage system into clearing process of multiple markets and enables such a facility to possibly affect the outcomes of those markets to its own benefit through strategic price and quantity offers and bids.

Over the past few years, a number of works studied the pool strategy of a merchant storage system in energy-only markets [4],[5],[6],[7], the joint energy and reserve markets as day-ahead settlement [2],[3], and the joint energy and reserve markets (day-ahead settlement) along with balancing settlement in real-time [8],[9],[10],[11].

In [4] and [5],[6], [7], the strategic decisions of a price-taker and a price-maker storage system are derived, respectively, all considering an energy-only market. Price-taker (price-maker) refers to an entity, whose operation decisions cannot (can) affect the market-clearing outcomes, i.e., clearing price and quantities. References [5],[6],[7] address a price-maker storage system that competes in an imperfectly competitive market.

References [2],[3] study the operation impacts of a pricetaker storage system in a day-ahead market as a joint energy and reserve settlement. However, the balancing stage is not modeled; therefore, the storage system has no knowledge on the amount of energy to be injected/consumed from its reserve in real-time under different foreseen scenarios. This makes the day-ahead decisions of the storage system less accurate since the real-time operation may alter its scheduling decisions in the day-ahead stage.

Finally, [8],[9],[10],[11] investigate the operation of a pricetaker storage system in a joint energy and reserve settlement (day-ahead market) considering the potential uncertainties in real-time. These works reflect the uncertainty in real-time using different techniques, e.g., a fixed energy deployment from committed reserve [8], a probabilistic distribution [9], robust method [10], and a set of scenarios [11]. In [9], the balancing operation does not impact the day-ahead strategic decisions. In [11], for the sake of computational tractability, an additional constraint linking energy and reserve is enforced within the problem.

A common assumption in [2],[3],[4],[8],[9],[10],[11] is that the storage system is price-taker. Although this assumption holds true for small-size facilities, it may not be necessarily the case for relatively large-scale storage systems [5]. Compared to [2],[3],[4],[8],[9],[10], [11], we model a price-maker storage system capable of capturing opportunities made by the bidirection impacts of the storage system's operation and market conditions. On the other hand, in studies which model storage system as a price-maker facility [5],[6],[7], storage system's decisions are made in an energy-only market. Considering high integration of wind power, an important observation is that the large-scale storage systems tend to offer strategically in the ancillary services markets, in particular reserve. Compared to [5],[6],[7], the proposed model in this paper captures the interactions of different markets and their impacts on the functioning of the storage system.

In this paper, we propose a decision-making tool based on stochastic bilevel modeling to determine the pool strategy of a price-maker storage system in day-ahead (as joint energy and reserve settlement) and balancing markets, while considering the net load uncertainty. The storage system behaves strategically within all markets. Note that the storage system bids (offers) strategically in its charging (discharging) mode in terms of energy price, energy quantity, and reserve quantity. The interaction among energy, reserve, and balancing markets and the decisions' dependency in these markets make an opportunity for the storage system to raise its expected profit. The final resulting model is a mixed-integer linear programming (MILP) problem, which can be solved using the available commercial software.

Note that some previous works (e.g., [12],[13]) have addressed the operation scheduling of a storage system where the facility is considered as a network asset. That is not the focus of the present work; rather, we focus on a merchant, for-profit facility.

The presented model takes into account the limitations of the storage system, the complexity and competition in the market, and the opportunities that exist for stacking multiple revenue streams. The developed model quantifies how a merchant storage system can increase its expected profit by optimally participating in multiple trading floors (such as dayahead and real-time balancing stages) and producing different market products (such as energy and reserve).

The rest of this paper is organized as follows. The mathematical formulation of the proposed model is provided in Section III The results are presented and discussed in Section III. Finally, the paper is concluded in Section IV. 


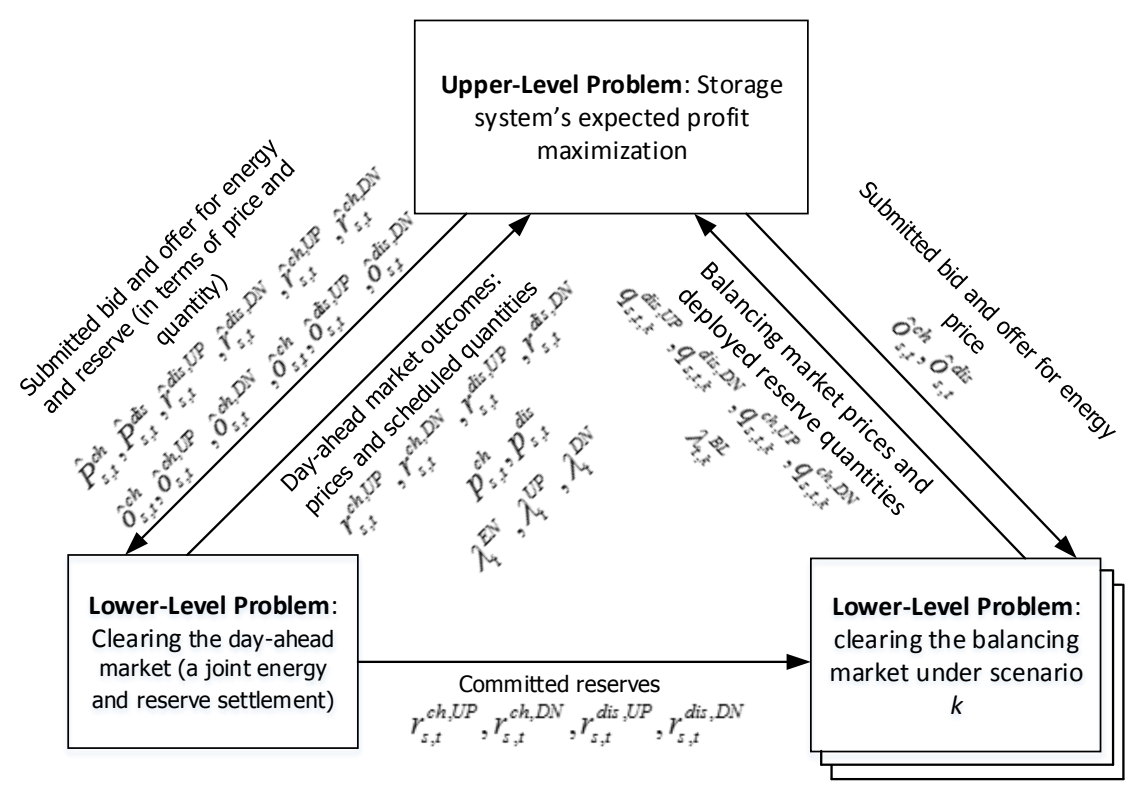

Fig. 1. The bilevel structure of the proposed complementarity model

\section{Methodology AND Formulation}

The proposed model is a bilevel programming problem, which is in fact a type of Stackelberg game with a single leader and one or more followers. Specifically, in this paper, the strategic storage is the leader, whereas the followers are clearing problems of day-ahead and balancing markets. This hierarchical structure is illustrated in Fig. 1. This problem is solved by the storage system. The leader (storage) makes its offering decisions in the upper level while anticipating the consequences of such decisions within the market-clearing problems as followers in the lower levels. This way, the storage system simultaneously determines its optimal participation strategy in both day-ahead and balancing markets.

The offering decisions of the storage system include its charging bids and discharging offers. For given storage system's decisions, the followers maximize market's social welfare through dispatch decisions. One of the lower-level problems represents the day-ahead joint energy and reserve market. The other set of lower-level problems (one per scenario) represents the real-time balancing market. This setup is similar to the structure of Pennsylvania-New Jersey-Maryland (PJM) market [14], where the balancing operating reserve is responsible to offset the wind power uncertainty [15]. In this hierarchical optimization setup, note that each lower-level problem is a constraint for the upper-level problem.

It is worth mentioning that since this problem is solved from the storage system point of view, the offering decisions of other market parties (e.g., conventional generators and demands) are necessarily treated as parameters. In this paper, we consider them as perfectly known data. However, it is straightforward to take into account their potential uncertainty via additional scenarios, but at the cost of increased computational complexity. In case of considering all market participants as strategic, the problem is converted to an equilibrium problem with equilibrium constraints, which is the focus of our future works. In line with real-world electricity markets, the day- ahead and balancing settlements are cleared sequentially, in the sense that the day-ahead outcomes (energy schedules and reserve commitments) are fixed (exogenous parameters) inside balancing markets.

The proposed bilevel model in this paper may yield multiple solutions; each solution refers to a different offering strategy for the strategic storage system, but its expected profit across all potential solutions is identical. As long as the selected strategy yields the highest expected profit for the storage system, it has no preference in choosing a particular solution among those obtained. The readers interested in bilevel models and their applications to power systems are referred to [16].

\section{A. Upper-level Problem: Expected Profit Maximization}

The upper-level problem seeks to maximize the expected profit of the storage system from all markets, and formulated below by a.1 a.18. The primal variables of the upper-level problem are $\Xi_{U L}=$ $\left\{\widehat{p}_{s, t}^{\mathrm{ch}}, \widehat{p}_{s, t}^{\mathrm{dis}}, \widehat{r}_{s, t}^{\mathrm{dis}, \mathrm{UP}}, \widehat{r}_{s, t}^{\mathrm{dis}, \mathrm{DN}}, \widehat{r}_{s, t}^{\mathrm{ch}, \mathrm{UP}}, \widehat{r}_{s, t}^{\mathrm{ch}, \mathrm{DN}}, u_{s, t}^{\mathrm{ch}}, u_{s, t}^{\mathrm{dis}}, \widehat{o}_{s, t}^{\mathrm{ch}}\right.$, $\left.\widehat{o}_{s, t}^{\text {ch,UP }}, \widehat{o}_{s, t}^{\text {ch,DN }}, \widehat{o}_{s, t}^{\text {dis }}, \widehat{o}_{s, t}^{\text {dis, UP }}, \widehat{o}_{s, t}^{\text {dis, DN }}, e_{s, t}\right\}$ as well as all lower-level primal and dual variables, which will be defined later:

$$
\begin{aligned}
& \underset{\Xi_{U L}}{\operatorname{Max}} \sum_{t=1}^{N_{t}} \sum_{s=1}^{N_{s}}\left[\left[-\left(\lambda_{t}^{\mathrm{EN}}+M C_{s}^{\mathrm{ch}}\right) \cdot p_{s, t}^{\mathrm{ch}}+\left(\lambda_{t}^{\mathrm{EN}}-M C_{s}^{\mathrm{dis}}\right) \cdot p_{s, t}^{\mathrm{dis}}\right]\right. \\
& +\left[\lambda_{t}^{\mathrm{UP}} \cdot\left(r_{s, t}^{\mathrm{ch}, \mathrm{UP}}+r_{s, t}^{\mathrm{dis}, \mathrm{UP}}\right)+\lambda_{t}^{\mathrm{DN}} \cdot\left(r_{s, t}^{\mathrm{ch}, \mathrm{DN}}+r_{s, t}^{\mathrm{dis}, \mathrm{DN}}\right)\right] \\
& +\sum_{k=1}^{N_{k}} \Phi_{k}\left[\left(\lambda_{t, k}^{\mathrm{BL}}+M C_{s}^{\mathrm{ch}}\right) \cdot\left(q_{s, t, k}^{\mathrm{ch}, \mathrm{UP}}-q_{s, t, k}^{\mathrm{ch}, \mathrm{DN}}\right)\right. \\
& \left.\left.\quad+\left(\lambda_{t, k}^{\mathrm{BL}}-M C_{s}^{\mathrm{dis}}\right) \cdot\left(q_{s, t, k}^{\mathrm{dis}, \mathrm{UP}}-q_{s, t, k}^{\mathrm{dis}, \mathrm{DN}}\right)\right]\right]
\end{aligned}
$$

\section{Subject to:}

$$
\begin{aligned}
& u_{s, t}^{\mathrm{dis}}+u_{s, t}^{\mathrm{ch}} \leq 1 \quad \forall s, \forall t \\
& u_{s, t}^{\mathrm{ch}}, u_{s, t}^{\mathrm{dis}} \in\{0,1\} \quad \forall s, \forall t \\
& 0 \leq \widehat{p}_{s, t}^{\mathrm{dis}} \leq u_{s, t}^{\mathrm{dis}} \cdot P_{s}^{\mathrm{dis}} \forall s, \forall t
\end{aligned}
$$




$$
\begin{aligned}
& 0 \leq \widehat{r}_{s, t}^{\mathrm{dis}, \mathrm{UP}} \leq u_{s, t}^{\mathrm{dis}} \cdot R_{s}^{\mathrm{dis}, \mathrm{UP}} \forall s, \forall t \\
& 0 \leq \widehat{r}_{s, t}^{\mathrm{dis}, \mathrm{DN}} \leq u_{s, t}^{\mathrm{dis}} \cdot R_{s}^{\mathrm{dis}, \mathrm{DN}} \forall s, \forall t \\
& \widehat{p}_{s, t}^{\mathrm{dis}}+\widehat{r}_{s, t}^{\mathrm{dis}, \mathrm{UP}} \leq u_{s, t}^{\mathrm{dis}} \cdot P_{s}^{\mathrm{dis}} \forall s, \forall t \\
& \widehat{r}_{s, t}^{\mathrm{dis}, \mathrm{DN}}-\widehat{p}_{s, t}^{\mathrm{dis}} \leq 0 \forall s, \forall t \\
& 0 \leq \widehat{p}_{s, t}^{\mathrm{ch}} \leq u_{s, t}^{\mathrm{ch}} \cdot P_{s}^{\mathrm{ch}} \forall s, \forall t \\
& 0 \leq \widehat{r}_{s, t}^{\mathrm{ch}, \mathrm{UP}} \leq u_{s, t}^{\mathrm{ch}} \cdot R_{s}^{\mathrm{ch}, \mathrm{UP}} \forall s, \forall t \\
& 0 \leq \widehat{r}_{s, t}^{\mathrm{ch}, \mathrm{DN}} \leq u_{s, t}^{\mathrm{ch}} \cdot R_{s}^{\mathrm{ch}, \mathrm{DN}} \forall s, \forall t \\
& \widehat{p}_{s, t}^{\mathrm{ch}}+\widehat{r}_{s, t}^{\mathrm{ch}, \mathrm{DN}} \leq u_{s, t}^{\mathrm{ch}} \cdot P_{s}^{\mathrm{ch}} \forall \mathrm{s}, \forall \mathrm{t} \\
& \widehat{r}_{s, t}^{\mathrm{ch}, \mathrm{UP}}-\widehat{p}_{s, t}^{\mathrm{ch}} \leq 0 \quad \forall s, \forall t \\
& \widehat{o}_{s, t}^{\mathrm{ch}}, \widehat{o}_{s, t}^{\mathrm{ch}, \mathrm{UP}}, \widehat{o}_{s, t}^{\mathrm{ch}, \mathrm{DN}} \geq 0 \quad \forall s, \forall t \\
& \widehat{o}_{s, t}^{\mathrm{dis}}, \widehat{o}_{s, t}^{\mathrm{dis}, \mathrm{UP}}, \widehat{o}_{s, t}^{\mathrm{dis}, \mathrm{DN}} \geq 0 \quad \forall s, \forall t \\
& 0 \leq e_{s, t} \leq E_{s} \forall s, \forall t \\
& e_{s, t}=E_{s}^{\mathrm{ini}}-\sum_{h=1}^{t}\left[p_{s, t}^{\mathrm{dis}}+\sum_{k=1}^{N_{k}} \Phi_{k}\left(q_{s, t, k}^{\mathrm{dis}, \mathrm{UP}}-q_{s, t, k}^{\mathrm{dis}, \mathrm{DN}}\right)\right] \\
& +\sum_{h=1}^{t} \eta_{s} \cdot\left[p_{s, t}^{\mathrm{ch}}+\sum_{k=1}^{N_{k}} \Phi_{k}\left(-q_{s, t, k}^{\mathrm{ch}, \mathrm{UP}}+q_{s, t, k}^{\mathrm{ch}, \mathrm{DN}}\right)\right] \quad \forall s, \forall t \\
& e_{s, t}=\alpha_{s} \cdot E_{s}^{\mathrm{ini}} \forall s, t=N_{t} .
\end{aligned}
$$

The objective function a.1 consists of profit sources from energy and reserve commitment (upward and downward) in day-ahead market, along with deployed energy from reserve in balancing market. Each profit source is envisioned within both operating modes, i.e., charging and discharging. Note that the hourly energy prices (i.e., $\lambda_{t}^{\mathrm{EN}}$ ), upward and downward reserve capacity commitment prices (i.e., $\lambda_{t}^{\mathrm{UP}}$ and $\lambda_{t}^{\mathrm{DN}}$ ), scheduled energy quantities (i.e., $p_{s, t}^{\text {ch }}$ and $p_{s, t}^{\text {dis }}$ ) and committed reserve quantities (i.e., $r_{s, t}^{\mathrm{ch}, \mathrm{UP}}, r_{s, t}^{\mathrm{ch}, \mathrm{DN}}, r_{s, t}^{\mathrm{dis}, \mathrm{UP}}$ and $r_{s, t}^{\mathrm{dis}, \mathrm{DN}}$ ) are endogenously obtained from the lower-level problem representing the clearing of day-ahead market. Similarly, the balancing market-clearing prices (i.e., $\lambda_{t, k}^{\mathrm{BL}}$ ) and the deployed reserves (i.e., $q_{s, t, k}^{\mathrm{ch}, \mathrm{UP}}, q_{s, t, k}^{\mathrm{ch}, \mathrm{DN}}, q_{s, t, k}^{\mathrm{dis}, \mathrm{UP}}$ and $q_{s, t, k}^{\text {dis,DN }}$ ) are endogenously derived from the lower-level problems representing the clearing of balancing market under different net load scenarios.

The first two lines in a.1 are associated with storage system's profit in the day-ahead market. The first line includes the cost of charging energy into the storage and the revenue from energy discharged. In addition, the second line in a.1 contains the storage system's revenue from upward and downward reserve capacity provision. The last two lines in a.1 are associated with the storage system's profit/cost in the balancing market, particularly in charging (third line) and discharging (fourth line) modes. In the charging mode (third line), the storage system is paid based on balancing market price (i.e., $\lambda_{t, k}^{\mathrm{BL}}$ ) if it is called for reducing its charging power by $q_{s, t, k}^{\text {ch,UP }}$ (upward reserve provision). In this case, the storage system earns $q_{s, t, k}^{\mathrm{ch}, \mathrm{UP}} \cdot\left(\lambda_{t, k}^{\mathrm{BL}}+M C_{s}^{\mathrm{ch}}\right)$ since it is also not incurred by the marginal cost (i.e., $M C_{s}^{\mathrm{ch}}$ ). In the same mode; however, the storage system is charged by $q_{s, t, k}^{\mathrm{ch}, \mathrm{DN}} \cdot\left(\lambda_{t, k}^{\mathrm{BL}}+\right.$ $\left.M C_{s}^{\mathrm{ch}}\right)$ due to extra power charged in the balancing stage, i.e., $q_{s, t, k}^{\mathrm{ch}, \mathrm{DN}}$ (downward reserve provision). Likewise, in the discharging mode (fourth line), the storage system injects power $q_{s, t, k}^{\text {dis, UP }}$ (upward reserve provision), and earns based on the balancing market price minus the cost of operation in the discharging mode (i.e., $\left.q_{s, t, k}^{\text {dis, UP }} \cdot\left(\lambda_{t, k}^{\mathrm{BL}}-M C_{s}^{\text {dis }}\right)\right)$. Finally, the storage system's cost in the discharging mode for downward reserve deployment is $q_{s, t, k}^{\mathrm{dis}, \mathrm{DN}} \cdot\left(\lambda_{t, k}^{\mathrm{BL}}-M C_{s}^{\mathrm{dis}}\right)$ since it pays back to the market at the balancing market price (i.e., $\lambda_{t, k}^{\mathrm{BL}}$ ) for the energy not produced, while saving the marginal cost.

The operation modes for the storage system are considered in a.2 and a.3. Constraint a.4, a.5 and a.6 restrict the energy and reserve quantity offers of storage system in its discharging mode. Constraints a.7 and a.8 limit those quantity offers as a whole to the discharging capacity. Constraints a.9-a.13 are similar to a.4 a.8 but in charging mode. Constraints a.14 and a.15 enforce the non-negativity of bidding and offering prices, either in terms of energy or reserve. Constraint a.16 enforces the reservoir capacity. Constraint a.17) represents the storage system's expected state of charge. Finally, constraint a.18 is designed to specify the storage system's state of charge at the end of the operation horizon. Note that constraints a.16-a.18 fulfill the storage requirements in expectation but not necessarily for each individual scenario. A generalization that fulfills the storage requirements by scenario is mathematically straightforward but at the cost of i) additional computational burden as it increases the number of constraints especially in cases with many scenarios, and ii) inefficient bidding strategy. In other words, the maximum profitability cannot be in general achieved while ensuring the fulfillment of all physical requirements by scenario. A potential future work may analyze how the storage system makes a trade-off between profitability and fulfillment of physical requirements by scenario. One potential approach is to incorporate the chance constraints [17] into the offering strategy problem. This way, the storage system can restrict the number of scenarios with unsatisfied physical requirements.

In case of a price-taker storage system [2],[3],[4],[8],[9],[10],[11], all market prices (i.e., $\lambda_{t}^{\mathrm{EN}}$, $\left.\lambda_{t}^{\mathrm{UP}}, \lambda_{t}^{\mathrm{DN}}, \lambda_{t, k}^{\mathrm{BL}}\right)$ are fixed parameters; therefore, the storage system solves a self-scheduling problem to determine its most beneficial charging and discharging actions for given prices. The resulting optimization problem is in general simpler than the proposed bilevel model in this paper for a strategic storage.

Note that the upper-level problem a.1 a.18 is constrained by lower-level problem (b.1)-(b.22) representing the clearing of day-ahead market, and lower-level problems (c.1)-(c.11) representing the clearing of balancing market under different scenarios. Such lower-level problems are presented in the next two sections. Note that all offering and bidding decisions of the storage system (all symbols with a hat, e.g., $\widehat{o}_{s, t}^{\text {ch }}$ ) are variables in the upper-level problem, but treated as parameters in the lower-level problems. This enables the storage system to gain insight into the market-clearing outcomes as a function of its offering and bidding decisions, and then adjust them in the upper-level problem pursuing expected profit maximization. 
B. Lower-Level Problem: Clearing of Day-Ahead Market (Joint Energy and Reserve Settelment)

The lower-level problem representing the clearing of dayahead market is given by (b.1)-(b.22) below. All dual variables are given in constraints after a colon. Variable set $\Xi_{L L-b}^{\text {Primal }}=$ $\left\{p_{s, t}^{\mathrm{ch}}, p_{s, t}^{\mathrm{dis}}, p_{d, t}, p_{g, t}, r_{s, t}^{\mathrm{ch}, \mathrm{UP}}, r_{s, t}^{\mathrm{ch}, \mathrm{DN}}, r_{s, t}^{\mathrm{dis}, \mathrm{UP}}, r_{s, t}^{\mathrm{dis}, \mathrm{DN}}, r_{g, t}^{\mathrm{UP}}\right.$ $\left., r_{g, t}^{\mathrm{DN}}, r_{d, t}^{\mathrm{UP}}, r_{d, t}^{\mathrm{DN}}\right\}$ contains the primal variables of the lower-level problem (b), whereas the dual variables of such a problem are included in the variable set $\Xi_{L L-b}^{\text {Dual }}=$ $\left\{\lambda_{t}^{\mathrm{EN}}, \lambda_{t}^{\mathrm{UP}}, \lambda_{t}^{\mathrm{DN}}, \underline{\mu}_{g, t}, \bar{\mu}_{g, t}, \underline{\mu}_{d, t}, \bar{\mu}_{d, t}, \underline{\mu}_{s, t}^{\mathrm{ch}}, \bar{\mu}_{s, t}^{\mathrm{ch}}, \underline{\mu}_{s, t}^{\mathrm{dis}}, \bar{\mu}_{s, t}^{\mathrm{dis}}\right.$ $, \underline{\mu}_{s, t}^{\mathrm{ch}, \mathrm{UP}}, \bar{\mu}_{s, t}^{\mathrm{ch}, \mathrm{UP}}, \underline{\mu}_{s, t}^{\mathrm{ch}, \mathrm{DN}}, \bar{\mu}_{s, t}^{\mathrm{ch}, \mathrm{DN}}, \underline{\mu}_{s, t}^{\mathrm{dis}, \mathrm{UP}}, \bar{\mu}_{s, t}^{\mathrm{dis}, \mathrm{UP}}, \underline{\mu}_{s, t}^{\mathrm{dis}, \mathrm{DN}}$ $\bar{\mu}_{s, t}^{\text {dis,DN }}, \mu_{s, t}^{\text {ch,UP }}, \mu_{s, t}^{\text {dis,DN }}, \mu^{\mathrm{UP}}, \bar{\mu}_{g, t}^{\mathrm{UP}}, \mu_{g, t}^{\mathrm{DN}}, \bar{\mu}_{g, t}^{\mathrm{DN}}, \mu^{\mathrm{UP}}$ $\left.\mu_{g, t}^{\mathrm{DN}}, \underline{\mu}_{d, t}^{\mathrm{UP}}, \bar{\mu}_{d, t}^{\mathrm{UP}}, \underline{\mu}_{d, t}^{\mathrm{DN}}, \bar{\mu}_{d, t}^{\mathrm{DN}}, \mu_{d, t}^{\mathrm{DN}}, \mu_{d, t}^{\mathrm{UP}}\right\}$.

$$
\begin{aligned}
& \left\{\underset{\Xi_{L L-b}^{\mathrm{Primal}}}{\operatorname{Max}} \sum_{s=1}^{N_{s}}\left(\widehat{o}_{s, t}^{\mathrm{ch}} \cdot p_{s, t}^{\mathrm{ch}}-\widehat{o}_{s, t}^{\mathrm{dis}} \cdot p_{s, t}^{\mathrm{dis}}\right)+\sum_{d=1}^{N_{d}} U_{d, t}^{\mathrm{EN}} \cdot p_{d, t}\right. \\
& -\sum_{g=1}^{N_{g}} O_{g, t}^{\mathrm{EN}} \cdot p_{g, t}-\sum_{s=1}^{N_{s}}\left(\widehat{o}_{s, t}^{\mathrm{ch}, \mathrm{UP}} \cdot r_{s, t}^{\mathrm{ch}, \mathrm{UP}}+\widehat{o}_{s, t}^{\mathrm{ch}, \mathrm{DN}} \cdot r_{s, t}^{\mathrm{ch}, \mathrm{DN}}\right. \\
& \left.+\widehat{o}_{s, t}^{\mathrm{dis}, \mathrm{UP}} \cdot r_{s, t}^{\mathrm{dis}, \mathrm{UP}}+\widehat{o}_{s, t}^{\mathrm{dis}, \mathrm{DN}} \cdot r_{s, t}^{\mathrm{dis}, \mathrm{DN}}\right) \\
& -\sum_{g=1}^{N_{g}} O_{g, t}^{\mathrm{RS}} \cdot\left(r_{g, t}^{\mathrm{UP}}+r_{g, t}^{\mathrm{DN}}\right)-\sum_{d=1}^{N_{d}} U_{d, t}^{\mathrm{RS}} \cdot\left(r_{d, t}^{\mathrm{UP}}+r_{d, t}^{\mathrm{DN}}\right)
\end{aligned}
$$

\section{Subject to:}

$$
\begin{aligned}
& \sum_{d=1}^{N_{d}} p_{d, t}-\sum_{s=1}^{N_{s}}\left(p_{s, t}^{\mathrm{dis}}-p_{s, t}^{\mathrm{ch}}\right)-\sum_{g=1}^{N_{g}} p_{g, t}=0 \quad: \lambda_{t}^{\mathrm{EN}} \\
& \sum_{s=1}^{N_{s}}\left(r_{s, t}^{\mathrm{ch}, \mathrm{UP}}+r_{s, t}^{\mathrm{dis}, \mathrm{UP}}\right)+\sum_{g=1}^{N_{g}} r_{g, t}^{\mathrm{UP}}+\sum_{d=1}^{N_{d}} r_{d, t}^{\mathrm{UP}}=R_{t}^{\mathrm{UP}}: \lambda_{t}^{\mathrm{UP}} \\
& \sum_{s=1}^{N_{s}}\left(r_{s, t}^{\mathrm{ch}, \mathrm{DN}}+r_{s, t}^{\mathrm{dis}, \mathrm{DN}}\right)+\sum_{g=1}^{N_{g}} r_{g, t}^{\mathrm{DN}}+\sum_{d=1}^{N_{d}} r_{d, t}^{\mathrm{DN}}=R_{t}^{\mathrm{DN}}: \lambda_{t}^{\mathrm{DN}} \\
& 0 \leq p_{g, t} \leq P_{g}: \underline{\mu}_{g, t}, \bar{\mu}_{g, t} ; \quad \forall g \\
& 0 \leq p_{d, t} \leq P_{d, t}: \underline{\mu}_{d, t}, \bar{\mu}_{d, t} ; \quad \forall d \\
& 0 \leq p_{s, t}^{\mathrm{ch}} \leq \widehat{p}_{s, t}^{\mathrm{ch}}: \underline{\mu}_{s, t}^{\mathrm{ch}}, \bar{\mu}_{s, t}^{\mathrm{ch}} ; \quad \forall s \\
& 0 \leq p_{s, t}^{\text {dis }} \leq \widehat{p}_{s, t}^{\text {dis }}: \underline{\mu}_{s, t}^{\text {dis }}, \bar{\mu}_{s, t}^{\text {dis }} ; \quad \forall s \\
& 0 \leq r_{s, t}^{\mathrm{ch}, \mathrm{UP}} \leq \widehat{r}_{s, t}^{\mathrm{ch}, \mathrm{UP}}: \underline{\mu}_{s, t}^{\mathrm{ch}, \mathrm{UP}}, \bar{\mu}_{s, t}^{\mathrm{ch}, \mathrm{UP}} ; \quad \forall s \\
& 0 \leq r_{s, t}^{\mathrm{ch}, \mathrm{DN}} \leq \widehat{r}_{s, t}^{\mathrm{ch}, \mathrm{DN}}: \underline{\mu}_{s, t}^{\mathrm{ch}, \mathrm{DN}}, \bar{\mu}_{s, t}^{\mathrm{ch}, \mathrm{DN}} ; \forall s \\
& 0 \leq r_{s, t}^{\mathrm{dis}, \mathrm{UP}} \leq \widehat{r}_{s, t}^{\mathrm{dis}, \mathrm{UP}}: \underline{\mu}_{s, t}^{\mathrm{dis}, \mathrm{UP}}, \bar{\mu}_{s, t}^{\mathrm{dis}, \mathrm{UP}} ; \quad \forall s \\
& 0 \leq r_{s, t}^{\mathrm{dis}, \mathrm{DN}} \leq \widehat{r}_{s, t}^{\mathrm{dis}, \mathrm{DN}}: \underline{\mu}_{s, t}^{\mathrm{dis}, \mathrm{DN}}, \bar{\mu}_{s, t}^{\mathrm{dis}, \mathrm{DN}} ; \quad \forall s \\
& r_{s, t}^{\mathrm{ch}, \mathrm{UP}} \leq p_{s, t}^{\mathrm{ch}}: \mu_{s, t}^{\mathrm{ch}, \mathrm{UP}} ; \quad \forall s \\
& r_{s, t}^{\mathrm{dis}, \mathrm{DN}} \leq p_{s, t}^{\mathrm{dis}}: \mu_{s, t}^{\mathrm{dis}, \mathrm{DN}} ; \forall s \\
& 0 \leq r_{g, t}^{\mathrm{UP}} \leq R_{g}^{\mathrm{UP}}: \underline{\mu}_{g, t}^{\mathrm{UP}}, \bar{\mu}_{g, t}^{\mathrm{UP}} ; \quad \forall g \\
& 0 \leq r_{g, t}^{\mathrm{DN}} \leq R_{g}^{\mathrm{DN}}: \underline{\mu}_{g, t}^{\mathrm{DN}}, \bar{\mu}_{g, t}^{\mathrm{DN}} ; \quad \forall g \\
& p_{g, t}+r_{g, t}^{\mathrm{UP}} \leq P_{g}: \mu_{g, t}^{\mathrm{UP}} ; \quad \forall g \\
& r_{g, t}^{\mathrm{DN}}-p_{g, t} \leq 0: \mu_{g, t}^{\mathrm{DN}} ; \quad \forall g \\
& 0 \leq r_{d, t}^{\mathrm{UP}} \leq R_{d, t}^{\mathrm{UP}}: \underline{\mu}_{d, t}^{\mathrm{UP}}, \bar{\mu}_{d, t}^{\mathrm{UP}} ; \quad \forall d \\
& 0 \leq r_{d, t}^{\mathrm{DN}} \leq R_{d, t}^{\mathrm{DN}}: \underline{\mu}_{d, t}^{\mathrm{DN}}, \bar{\mu}_{d, t}^{\mathrm{DN}} ; \quad \forall d
\end{aligned}
$$

$p_{d, t}+r_{d, t}^{\mathrm{DN}} \leq P_{d, t}: \mu_{d, t}^{\mathrm{DN}} ; \quad \forall d$

$r_{d, t}^{\mathrm{UP}}-p_{d, t} \leq 0: \mu_{d, t}^{\mathrm{UP}} ; \quad \forall d$

(b.22) $\} \forall t$

The objective function (b.1) maximizes the surplus in the day-ahead market, which consists of energy and reserve terms. For the sake of generality, upward and downward reserve requirements of the market can be provided by generators, demands, and storage system in its both operating modes.

Constraint b.2 enforces the energy balance, and its corresponding dual variable provides the energy price. Constraints (b.3) and b.4 enforce meeting the reserve requirements of the market from different resources, and their associated dual variables give the upward and downward reserve capacity commitment prices, respectively. Constraint b.5 restricts the energy production level of generators. Constraint (b.6) limits the consumption level of demands. Constraints (b.7) and (b.8) enforce the storage system to be charged and discharged between zero and its quantity bids and offers. Constraints (b.9)-(b.14) limit the reserve commitment of storage system. Similarly, the reserve commitments of generators and demands are restricted by (b.15)-(b.18) and (b.19)-(b.22), respectively. To be able to closely tract the results, we ignore the transmission network and generator's ramping constraints at this step of our work. Extending the model to include these constraints is straightforward [7] and is left to future work.

\section{Lower-Level Problems: Clearing of Balancing Market Un- der Different Net Load Deviation Scenarios}

The lower-level problems representing the clearing of balancing market under different net load deviation scenarios are given by (c.1)-(c.11). The primal variables of lower-level problems (c), one per scenario, are included in set $\Xi_{L L-c}^{\text {Primal }}=$ $\left\{l_{d, t, k}, q_{g, t, k}^{\mathrm{UP}}, q_{g, t, k}^{\mathrm{DN}}, q_{s, t, k}^{\mathrm{dis}, \mathrm{UP}}, q_{s, t, k}^{\mathrm{dis}, \mathrm{DN}}, q_{s, t, k}^{\mathrm{ch}, \mathrm{UP}}, q_{s, t, k}^{\mathrm{ch}, \mathrm{DN}}, q_{d, t, k}^{\mathrm{UP}}\right.$

$\left.q_{d, t, k}^{\mathrm{DN}}\right\}$ while the dual variables of such lower-level problems are within $\Xi_{L L-c}^{\text {Dual }}=$ $\left\{\lambda_{t, k}^{\mathrm{BL}}, \underline{\rho}_{g, t, k}^{\mathrm{UP}}, \bar{\rho}_{g, t, k}^{\mathrm{UP}}, \underline{\rho}_{g, t, k}^{\mathrm{DN}}, \bar{\rho}_{g, t, k}^{\mathrm{DN}}, \underline{\rho}_{d, t, k}^{\mathrm{UP}}\right.$,

$\bar{\rho}_{d, t, k}^{\mathrm{UP}}, \rho_{s, t, k}^{\mathrm{dis}, \mathrm{UP}}, \bar{\rho}_{s, t, k}^{\mathrm{dis}, \mathrm{UP}}, \rho_{s, t, k}^{\mathrm{dis}, \mathrm{DN}}, \bar{\rho}_{s, t, k}^{\mathrm{dis}, \mathrm{DN}}, \rho_{s, t, k}^{\mathrm{ch}, \mathrm{UP}}, \bar{\rho}_{s, t, k}^{\mathrm{ch}, \mathrm{UP}}$ $\left.\underline{\rho}_{s, t, k}^{\mathrm{ch}, \mathrm{DN}}, \bar{\rho}_{s, t, k}^{\mathrm{ch}, \mathrm{DN}}, \underline{\rho}_{d, t, k}, \bar{\rho}_{d, t, k}\right\}$.

$$
\begin{aligned}
& \left\{\underset{\substack{\Xi_{L L-c}^{\mathrm{Prim}} \\
\operatorname{Min}_{L}}}{ } \sum_{d=1}^{N_{d}} V_{d, t} \cdot l_{d, t, k}+\sum_{g=1}^{N_{g}} O_{g, t}^{\mathrm{EN}} \cdot\left(q_{g, t, k}^{\mathrm{UP}}-q_{g, t, k}^{\mathrm{DN}}\right)\right. \\
& +\sum_{s=1}^{N_{s}} \widehat{o}_{s, t}^{\mathrm{dis}} \cdot\left(q_{s, t, k}^{\mathrm{dis}, \mathrm{UP}}-q_{s, t, k}^{\mathrm{dis}, \mathrm{DN}}\right)+\sum_{s=1}^{N_{s}} \widehat{o}_{s, t}^{\mathrm{ch}} \cdot\left(q_{s, t, k}^{\mathrm{ch}, \mathrm{UP}}-q_{s, t, k}^{\mathrm{ch}, \mathrm{DN}}\right) \\
& +\sum_{d=1}^{N_{d}} U_{d, t}^{\mathrm{EN}} \cdot\left(q_{d, t, k}^{\mathrm{UP}}-q_{d, t, k}^{\mathrm{DN}}\right)
\end{aligned}
$$

\section{Subject to:}

$$
\begin{aligned}
& \sum_{d=1}^{N_{d}} l_{d, t, k}+\sum_{s=1}^{N_{s}}\left(q_{s, t, k}^{\mathrm{ch}, \mathrm{UP}}-q_{s, t, k}^{\mathrm{ch}, \mathrm{DN}}\right)+\sum_{s=1}^{N_{s}}\left(q_{s, t, k}^{\mathrm{dis}, \mathrm{UP}}-q_{s, t, k}^{\mathrm{dis}, \mathrm{DN}}\right) \\
& +\sum_{g=1}^{N_{g}}\left(q_{g, t, k}^{\mathrm{UP}}-q_{g, t, k}^{\mathrm{DN}}\right)+\sum_{d=1}^{N_{d}}\left(q_{d, t, k}^{\mathrm{UP}}-q_{d, t, k}^{\mathrm{DN}}\right)=Q_{t, k}: \lambda_{t, k}^{\mathrm{BL}} \\
& 0 \leq q_{g, t, k}^{\mathrm{UP}} \leq r_{g, t}^{\mathrm{UP}}: \underline{\rho}_{g, t, k}^{\mathrm{UP}}, \bar{\rho}_{g, t, k}^{\mathrm{UP}} ; \quad \forall g \\
& 0 \leq q_{g, t, k}^{\mathrm{DN}} \leq r_{g, t}^{\mathrm{DN}}: \underline{\rho}_{g, t, k}^{\mathrm{DN}}, \bar{\rho}_{g, t, k}^{\mathrm{DN}} ; \quad \forall g
\end{aligned}
$$




$$
\begin{aligned}
& 0 \leq q_{d, t, k}^{\mathrm{UP}} \leq r_{d, t}^{\mathrm{UP}}: \underline{\rho}_{d, t, k}^{\mathrm{UP}}, \bar{\rho}_{d, t, k}^{\mathrm{UP}} ; \quad \forall d \\
& 0 \leq q_{d, t, k}^{\mathrm{DN}} \leq r_{d, t}^{\mathrm{DN}}: \underline{\rho}_{d, t, k}^{\mathrm{DN}}, \bar{\rho}_{d, t, k}^{\mathrm{DN}} ; \quad \forall d \\
& 0 \leq q_{s, t, k}^{\mathrm{dis}, \mathrm{UP}} \leq r_{s, t}^{\mathrm{dis}, \mathrm{UP}}: \underline{\rho}_{s, t, k}^{\mathrm{dis}, \mathrm{UP}}, \bar{\rho}_{s, t, k}^{\mathrm{dis}, \mathrm{UP}} ; \quad \forall s \\
& 0 \leq q_{s, t, k}^{\mathrm{dis}, \mathrm{DN}} \leq r_{s, t}^{\mathrm{dis}, \mathrm{DN}}: \underline{\rho}_{s, t, k}^{\mathrm{dis}, \mathrm{DN}}, \bar{\rho}_{s, t, k}^{\mathrm{dis}, \mathrm{DN}} ; \quad \forall s \\
& 0 \leq q_{s, t, k}^{\mathrm{ch}, \mathrm{UP}} \leq r_{s, t}^{\mathrm{ch}, \mathrm{UP}}: \underline{\rho}_{s, t, k}^{\mathrm{ch}, \mathrm{UP}}, \bar{\rho}_{s, t, k}^{\mathrm{ch}, \mathrm{UP}} ; \quad \forall s \\
& 0 \leq q_{s, t, k}^{\mathrm{ch}, \mathrm{DN}} \leq r_{s, t}^{\mathrm{ch}, \mathrm{DN}}: \underline{\rho}_{s, t, k}^{\mathrm{ch}, \mathrm{DN}}, \bar{\rho}_{s, t, k}^{\mathrm{ch}, \mathrm{DN}} ; \quad \forall s \\
& \left.0 \leq l_{d, t, k} \leq p_{d, t}: \underline{\rho}_{d, t, k}, \bar{\rho}_{d, t, k} ; \quad \forall d \quad \text { (c.11) }\right\} \forall t, \forall k .
\end{aligned}
$$

The main reason for net load deviation is the variable generation. We assume that generators with variable production (e.g., wind power producers) offer their production to dayahead and real-time markets at zero price, and therefore, they are treated as negative loads. Based on available variable generation forecast in day ahead, the elastic demands bid to day-ahead market only that part of their loads that is not supplied by zero-cost variable generation. However, the variable generation forecast in day ahead might not be accurate, and therefore, the potential mismatch (represented by parameter $\left.Q_{t, k}\right)$ needs to be offset in the balancing stage.

The objective function c.1 minimizes the social imbalance cost (equivalent to the minus social surplus) incurred by the net load deviation. The first term represents the involuntarily load shedding cost. The second term includes the production cost of conventional generators for energy deployment from their committed upward reserve, while those generators pay back to the market operator due to reducing their production (downward reserve provision). The third term of (c.1) is similar to the second term, but it corresponds to the storage systems in the discharging mode. The fourth and fifth terms of c.1 represent the costs/utilities of storage systems (in charging mode) and demands, respectively. These two terms represent the cost of demands and storage systems in charging mode for providing upward reserve (i.e., consumption reduction), as well as their utility obtained by extra consumption (downward reserve provision).

Constraint c.2 enforces offsetting the net load deviation, and its dual variable provides the balancing market price. A positive value for parameter $Q_{t, k}$ implies the need for extra power at time $t$ under scenario $k$, which can be provided by committed upward reserves by generators, demands, storage systems (in both modes) and involuntarily load curtailment. Similarly, a negative value for parameter $Q_{t, k}$ indicates the need for downward reserve resources.

Constraints c.3 -c.10 restrict the energy deployed from each available reserve resource based on its commitment in day-ahead market. Finally, constraint (c.11) limits the involuntary curtailed load.

\section{Solution Technique}

The solution technique of the bilevel model (a)-(c) is explained as follows: we first replace the lower-level problems (b) and (c) by their Karush-Kuhn-Tucker (KKT) conditions, as given in Appendix A. Note that these KKT conditions provide the optimality conditions since the lower-level problems (b) and (c) are linear and continuous. The resulting single-level optimization model is a mathematical program with equilibrium constraints (MPEC). This model, however, is non-linear. There are two sources of nonlinearity that can be linearized as described below:

- The first source of non-linearity is complementarity conditions d.13)-(d.42) and (e.10)-(e.27), which are within the KKT conditions. Each complementarity condition at the form of $0 \leq c \perp d \geq 0$ can be readily linearized without approximation using a Big-M approach [18] but at the cost of introducing a set of auxiliary binary variables. The resulting mixed-integer conditions are $c \geq 0, d \geq 0$, $c \leq u . M$, and $d \leq(1-u) . M$. Note that $u$ is an auxiliary binary variable and $M$ is a large enough positive constant. The selection of an appropriate value for $M$ could be a challenge, because a too big value may not hold the complementarity condition, while a too small value may result in numerical ill-conditioning and/or increase drastically the computational time. In this paper, for each of complementarity conditions linearized, we first arbitrarily choose a big value for $M$, e.g., $10^{7}$, and then solve the model. In the next step, we check the results to investigate whether each of the complementarity conditions holds. If not, we reduce the value of corresponding $M$ until all complementarity conditions are satisfied.

- The second source of non-linearity comes from the bilinear terms in objective function (a.1); each one is a product of price and quantity (either energy or reserve). Inspired from [19], we linearize without approximation most of those bilinear terms as described in Appendix B. The remained bilinear terms are also linearized (but with approximation) using a binary expansion method [20] as explained in Appendix B.

\section{NUMERICAL RESULTS}

In this section, we investigate the performance of our presented model using an illustrative example (Section III.A) and a real-world case study based on Alberta electricity market (Section III.B.) We solve the resulting MILP model using CPLEX under GAMS on an Intel(R) Core(TM) i7-5930K with 64 GB of RAM.

\section{A. Illustrative Test System}

This section provides an illustrative test system to demonstrate the performance of the proposed trading decisionmaking tool for an energy storage system. In this test system, a daily (24 hours) time horizon is considered. Technical characteristics of storage system and generators are given in Tables I and II respectively. Inspired by compressed air energy storage systems, we model separate charging and discharging devices [2]. The hourly loads are given in the second column of Table III. We assume that loads bid their energy demands across the whole time horizon at an identical price, i.e., $\$ 400 / \mathrm{MWh}$. The hourly maximum reserve capacity of demand is considered to be $10 \%$ of its load. The following cases are studied and compared: 
TABLE I

DATA FOR THE STORAGE SYSTEM

\begin{tabular}{|c|c|c|c|c|c|c|c|c|c|c|}
\hline $\begin{array}{l}\text { Storage } \\
\text { (s) }\end{array}$ & $\begin{array}{l}P_{s}^{\text {dis }} \\
\text { (MW) }\end{array}$ & $\begin{array}{l}P_{s}^{\mathrm{ch}} \\
(\mathrm{MW})\end{array}$ & $\begin{array}{l}M C_{s}^{\text {dis }} \\
\text { (\$/MWh) }\end{array}$ & $\begin{array}{c}M C_{s}^{\mathrm{ch}} \\
\text { (\$/MWh) }\end{array}$ & $\begin{array}{c}R_{s}^{\mathrm{ch}, \mathrm{UP}}=R_{s}^{\mathrm{ch}, \mathrm{DN}} \\
(\mathrm{MW})\end{array}$ & $\begin{array}{c}R_{s}^{\mathrm{dis}, \mathrm{UP}}=R_{s}^{\mathrm{dis}, \mathrm{DN}} \\
(\mathrm{MW})\end{array}$ & $\begin{array}{c}E_{s} \\
(\mathrm{MWh})\end{array}$ & $\begin{array}{c}E_{s}^{\text {ini }} \\
(\mathrm{MWh})\end{array}$ & $\alpha_{s}$ & $\eta_{s}$ \\
\hline Illustrative Test System & 20 & 15 & 20 & 2 & 10 & 10 & 120 & 0 & 1 & 1 \\
\hline Alberta Case & 100 & 50 & 12 & 2 & 100 & 50 & 400 & 0 & 1 & 0.5 \\
\hline
\end{tabular}

TABLE II

DATA FOR GENERATORS (ILLUSTRATIVE TEST SYSTEM)

\begin{tabular}{cccccc}
\hline Unit No. & $\begin{array}{c}P_{g} \\
(\mathrm{MW})\end{array}$ & $\begin{array}{c}O_{g, t}^{\mathrm{EN}} \\
(\$ / \mathrm{MWh})\end{array}$ & $\begin{array}{c}O_{g, t}^{\mathrm{RS}} \\
(\$ / \mathrm{MWh})\end{array}$ & $\begin{array}{c}R_{g}^{\mathrm{UP}} \\
(\mathrm{MW})\end{array}$ & $\begin{array}{c}R_{g}^{\mathrm{DN}} \\
(\mathrm{MW})\end{array}$ \\
\hline G1 & 120 & 12 & 5 & 20 & 20 \\
G2 & 50 & 30 & 15 & 20 & 20 \\
G3 & 30 & 90 & 25 & 20 & 20 \\
G4 & 30 & 120 & 40 & 20 & 20 \\
\hline
\end{tabular}

TABLE III

THE STORAGE SYSTEM BIDDING AND OFFERING DECISIONS, AND MARKET-CLEARING OUTCOMES IN CASE 1

\begin{tabular}{|c|c|c|c|c|c|c|c|}
\hline \multirow[b]{2}{*}{ Hour } & \multirow[b]{2}{*}{$\begin{array}{c}P_{d, t} \\
(\mathrm{MW})\end{array}$} & \multicolumn{2}{|c|}{ Storage system } & \multicolumn{3}{|c|}{$p_{g, t}$} & \multirow{2}{*}{$\begin{array}{c}\lambda_{t}^{\mathrm{EN}} \\
(\$ / \mathrm{MWh})\end{array}$} \\
\hline & & $\begin{array}{c}p_{s, t}^{\mathrm{dis}} / p_{s, t}^{\mathrm{ch}} \\
(\mathrm{MW})^{*}\end{array}$ & $\begin{array}{l}\widehat{o}_{s, t}^{\mathrm{dis}} / \widehat{o}_{s, t}^{\mathrm{ch}} \\
(\$ / \mathrm{MWh})\end{array}$ & G1 & $\begin{array}{c}\text { G2 G3 } \\
\text { (MW) }\end{array}$ & G4 & \\
\hline 1 & 60 & -15 & 12 & 75 & & & 12 \\
\hline 2 & 80 & -15 & 12 & 95 & & & 12 \\
\hline 3 & 100 & -5 & 12 & 105 & & & 12 \\
\hline 4 & 80 & & & 80 & & & 12 \\
\hline 5 & 80 & & & 80 & & & 12 \\
\hline 6 & 60 & -15 & 12 & 75 & & & 12 \\
\hline 7 & 80 & & & 80 & & & 12 \\
\hline 8 & 100 & & & 100 & & & 12 \\
\hline 9 & 120 & & & 120 & & & 30 \\
\hline 10 & 100 & & & 100 & & & 12 \\
\hline 11 & 100 & & & 100 & & & 12 \\
\hline 12 & 80 & -15 & 12 & 95 & & & 12 \\
\hline 13 & 100 & -15 & 12 & 115 & & & 12 \\
\hline 14 & 120 & & & 120 & & & 30 \\
\hline 15 & 140 & & & 120 & 20 & & 30 \\
\hline 16 & 160 & & & 120 & 40 & & 30 \\
\hline 17 & 200 & 20 & 90 & 120 & 40 & & 90 \\
\hline 18 & 180 & 20 & 90 & 120 & 40 & & 90 \\
\hline 19 & 180 & 20 & 90 & 120 & 40 & & 90 \\
\hline 20 & 200 & 20 & 90 & 120 & 40 & & 90 \\
\hline 21 & 160 & & & 120 & 40 & & 30 \\
\hline 22 & 140 & & & 120 & 20 & & 30 \\
\hline 23 & 100 & & & 100 & & & 12 \\
\hline 24 & 80 & & & 80 & & & 12 \\
\hline
\end{tabular}

*A negative/positive value corresponds to the storage system's charging/discharging mode.

- Case 1: A sole energy-only day-ahead market is considered. The only profit source of the storage system is energy arbitrage over time.

- Case 2: The day-ahead market is in fact a joint energy and reserve settlement, but the balancing stage is still not considered. The storage system makes a profit from energy trading as well as reserve provision.

- Case 3: Both day-ahead (joint energy and reserve settlement) and balancing markets are considered. This is the only case among Cases 1 to 3 , which models the net load deviation uncertainty since the balancing stage is included. In this case, the storage system achieves profit from energy trading, reserve provision in dayahead, and energy deployed from the committed reserve in the balancing stage.

In Case 1, the storage system makes a profit through energy arbitrage, provided that the price difference between charging and discharging hours is higher than the total marginal costs of storage system's charging and discharging devices [5], which is $\$ 22 / \mathrm{MWh}$ in this case (Table If). As given in Table III. the storage system charges during low-demand hours in which cheap generator G1 is the only dispatched unit. In all charging hours, the storage system strategically bids at $\$ 12 / \mathrm{MWh}$, which is equal to G1's offer price; and therefore, the energy price is set to that price. Then, the storage system discharges during peak hours, and strategically offers at a price equal to the offer price of $\mathrm{G} 3$, which is the marginal producer. Therefore, energy price is $\$ 90 / \mathrm{MWh}$. One interesting observation is that the storage offers strategically to affect both market prices and quantities such that the highest possible profit achieves. For example in hour 17, the storage could offer at $\$ 120 / \mathrm{MWh}$, which is equal to G4's offer price. Such an offer would lead to an energy price of $\$ 120 / \mathrm{MWh}$, but energy schedule of storage would drop to $10 \mathrm{MW}$ (instead of $20 \mathrm{MW}$ in the current results). In this particular case, the lower energy dispatch reduces profit, though the energy price is higher. Therefore, the optimal offering price for the storage system is $\$ 90 / \mathrm{MWh}$, and not $\$ 120 / \mathrm{MWh}$. Note that considering the generator's submitted offers and storage system's marginal cost, the storage system can make money in four hours, i.e., hours 17-20. In these hours, the storage system discharges $80 \mathrm{MWh}$. The storage system charges this amount of energy when demand and consequently the market price is low. Note that in this example, the quantity offer/bid of the storage system in each hour (i.e., $\widehat{p}_{s, t}^{\text {ch }}, \widehat{p}_{s, t}^{\text {dis }}$ ) is always identical to the scheduled quantity in that hour (i.e., $\left.p_{s, t}^{\mathrm{ch}}, p_{s, t}^{\mathrm{dis}}\right)$. In other words, the quantity bids and offers of the storage system are fully accepted in the market.

In Case 2, the storage system trades in day-ahead market as a joint energy and reserve settlement. The results are given in Table IV] In line with [3], the storage system charges and discharges in a higher number of hours in Case 2 compared to Case 1 (energy-only case). This way, the storage system gets more opportunities to make a profit. The storage system bids to charge to a level of energy that is seen profitable when discharged. Thus, there is no reason storage charges more than that. Note that the upward and downward reserve prices are equal at the charging hours. However, offering upward reserve at the charging hours creates the opportunity for the storage system to submit higher quantity offers in the energy market.

In the joint energy and reserve settlement (Case 2), energy market prices are different than those in the energyonly settlement (Case 1). In this case, the storage system strategically bids and offers to take advantage of energy and reserve prices' dependency. One interesting observation is that the energy offer price of storage may not be identical to that of other generators. For example in hours 17 and 20, it offers to produce energy at price $\$ 105 / \mathrm{MWh}$, which is different than that of other generators. The energy prices in those two hours are also $\$ 105 / \mathrm{MWh}$. The reason behind this offer price is the interaction of energy and reserve markets [21]. To supply one additional MW in hours 17 and 20, the schedule of G3 increases to $21 \mathrm{MW}$, while its upward reserve commitment drops to $9 \mathrm{MW}$. To meet the reserve requirements of the market, the upward reserve commitment of G4 increases to $11 \mathrm{MW}$. Therefore, the cost for supply the additional demand is $\$ 90 / \mathrm{MWh}$ (G3's energy offer price) minus $\$ 25 / \mathrm{MWh}$ (G3's 
TABLE IV

THE STORAGE SYSTEM BIDDING AND OFFERING DECISIONS, AND MARKET-CLEARING OUTCOMES IN CASE 2

\begin{tabular}{|c|c|c|c|c|c|c|c|c|c|c|c|c|c|c|c|c|c|}
\hline \multirow{5}{*}{ Hr. } & \multirow{5}{*}{$\begin{array}{c}R_{t}^{\mathrm{DN}}, \\
R_{t}^{\mathrm{UP}} \\
\\
(\mathrm{MW})^{\dagger}\end{array}$} & \multicolumn{6}{|c|}{ Storage } & \multicolumn{8}{|c|}{ Generators } & \multirow{2}{*}{\multicolumn{2}{|c|}{ Market prices }} \\
\hline & & \multicolumn{2}{|c|}{ Energy } & \multicolumn{4}{|c|}{ Reserve } & \multirow{2}{*}{\multicolumn{4}{|c|}{$\begin{array}{c}\text { Energy } \\
p_{g, t}\end{array}$}} & \multirow{2}{*}{\multicolumn{4}{|c|}{$\begin{array}{c}\text { Reserve } \\
r_{g, t}^{D N}, r_{g, t}^{U P}\end{array}$}} & & \\
\hline & & \multirow{3}{*}{$\begin{array}{c}p_{s, t}^{\mathrm{dis}}, \\
p_{s, t}^{\mathrm{ch}} \\
(\mathrm{MW})^{*}\end{array}$} & \multirow{3}{*}{$\begin{array}{c}\widehat{o}_{s, t}^{\mathrm{dis}}, \\
\widehat{o}_{s, t}^{\mathrm{ch}} \\
(\$ / \mathrm{MWh})\end{array}$} & \multirow{3}{*}{$\begin{array}{l}r_{s, t}^{\mathrm{ch}, \mathrm{DN}}, \\
r_{s, t}^{\mathrm{ch}, \mathrm{UP}} \\
(\mathrm{MW}) \dagger\end{array}$} & \multirow{3}{*}{$\begin{array}{c}\widehat{o}_{s, t}^{\mathrm{ch}, \mathrm{DN}} \\
\widehat{o}_{s, t}^{\mathrm{ch}, \mathrm{UP}} \\
(\$ / \mathrm{MWh})\end{array}$} & \multirow{3}{*}{$\begin{array}{c}r_{s, t}^{\text {dis,DN }}, \\
r_{s, t}^{\text {dis,UP }} \\
(\mathrm{MW})^{\dagger}\end{array}$} & \multirow{3}{*}{$\begin{array}{c}\widehat{o}_{s, t}^{\text {dis,DN }}, \\
\widehat{o}_{s, t}^{\text {dis, UP }} \\
(\$ / \mathrm{MWh})\end{array}$} & & & & & & & & & \multirow{3}{*}{$\begin{array}{c}\lambda_{t}^{\mathrm{EN}} \\
(\$ / \mathrm{MWh})\end{array}$} & \multirow{3}{*}{$\begin{array}{c}\lambda_{t}^{\mathrm{DN}} \\
\lambda_{t}^{\mathrm{UP}} \\
(\$ / \mathrm{MWh})\end{array}$} \\
\hline & & & & & & & & G1 & G2 & G3 & G4 & G1 & $\mathrm{G} 2$ & G3 & G4 & & \\
\hline & & & & & & & & \multicolumn{4}{|c|}{ (MW) } & \multicolumn{4}{|c|}{$(\mathrm{MW})^{\dagger}$} & & \\
\hline 1 & $-6,6$ & -9 & 12 & $-6,6$ & 5,5 & & & 69 & & & & & & & & 12 & 5,5 \\
\hline 2 & $-8,8$ & -8 & 12 & $-7,8$ & 5,5 & & & 88 & & & & $-1,0$ & & & & 12 & 5,5 \\
\hline 3 & $-10,10$ & -10 & 12 & $-5,10$ & 5,5 & & & 110 & & & & $-5,0$ & & & & 12 & 5,5 \\
\hline 4 & $-8,8$ & -8 & 12 & $-7,8$ & 5,5 & & & 88 & & & & $-1,0$ & & & & 12 & 5,5 \\
\hline 5 & $-8,8$ & -8 & 12 & $-7,8$ & 5,5 & & & 88 & & & & $-1,0$ & & & & 12 & 5,5 \\
\hline 6 & $-6,6$ & -9 & 12 & $-6,6$ & 5,5 & & & 69 & & & & 0,0 & & & & 12 & 5,5 \\
\hline 7 & $-8,8$ & -8 & 12 & $-7,8$ & 5,5 & & & 88 & & & & $-1,0$ & & & & 12 & 5,5 \\
\hline 8 & $-10,10$ & -10 & 12 & $-5,10$ & 5,5 & & & 110 & & & & $-5,0$ & & & & 12 & 5,5 \\
\hline 9 & $-12,12$ & & & & & 0,10 & 15 & 120 & & & & $-12,0$ & 0,2 & & & 30 & 5,15 \\
\hline 10 & $-10,10$ & -10 & 12 & $-5,10$ & 5,5 & & & 110 & & & & $-5,0$ & & & & 12 & 5,5 \\
\hline 11 & $-10,10$ & -10 & 12 & $-5,10$ & 5,5 & & & 110 & & & & $-5,0$ & & & & 12 & 5,5 \\
\hline 12 & $-8,8$ & -8 & 12 & $-7,8$ & 5,5 & & & 88 & & & & $-1,0$ & & & & 12 & 5,5 \\
\hline 13 & $-10,10$ & -10 & 12 & $-5,10$ & 5,5 & & & 110 & & & & $-5,0$ & & & & 12 & 5,5 \\
\hline 14 & $-12,12$ & & & & & 0,10 & 15 & 120 & & & & $-12,0$ & 0,2 & & & 30 & 5,15 \\
\hline 15 & $-14,14$ & 10 & 30 & & & $-10,10$ & 5,15 & 120 & 10 & & & $-4,0$ & 0,4 & & & 30 & 5,15 \\
\hline 16 & $-16,16$ & 3 & 40 & & & $-3,10$ & 5,15 & 120 & 37 & & & $-13,0$ & 0,3 & 0,3 & & 40 & 5,25 \\
\hline 17 & $-20,20$ & 20 & 105 & & & $-10,0$ & 5,40 & 120 & 40 & 20 & & $-10,0$ & & 0,10 & 0,10 & 105 & 5,40 \\
\hline 18 & $-18,18$ & 20 & 90 & & & $-10,0$ & 5,25 & 120 & 40 & & & $-8,0$ & & 0,18 & & 90 & 5,25 \\
\hline 19 & $-18,18$ & 20 & 90 & & & $-10,0$ & 5,25 & 120 & 40 & & & $-8,0$ & & 0,18 & & 90 & 5,25 \\
\hline 20 & $-20,20$ & 20 & 105 & & & $-10,0$ & 5,40 & 120 & 40 & 20 & & $-10,0$ & & 0,10 & 0,10 & 105 & 5,40 \\
\hline 21 & $-16,16$ & 5 & 40 & & & $-5,10$ & 5,15 & 120 & 35 & & & $-11,0$ & 0,5 & 0,1 & & 40 & 5,25 \\
\hline 22 & $-14,14$ & 10 & 30 & & & $-10,10$ & 5,15 & 120 & 10 & & & $-4,0$ & 0,4 & & & 30 & 5,15 \\
\hline 23 & $-10,10$ & & & & & 0,10 & 5 & 100 & & & & $-10,0$ & & & & 12 & 5,5 \\
\hline 24 & $-8,8$ & & & $-8,0$ & & & 5 & 80 & & & & 0,8 & & & & 12 & 5,5 \\
\hline
\end{tabular}

$\dagger$ A negative/positive value corresponds to the downward/upward reserve.

* A negative/positive value corresponds to the storage system's charging/discharging mode.

reserve offer price) plus $\$ 40 / \mathrm{MWh}$ (G4's reserve offer price), which is $\$ 105 / \mathrm{MWh}$.

We also compare Case 2 referring to the joint energy and reserve markets with a case in which energy and reserve settlement are cleared sequentially [22], which is the case of most European electricity markets (the energy schedules are fixed within the reserve market). The hourly reserve commitments of storage system in those two market setups are compared in Fig. 2. Accordingly, the storage system submits offers for a higher number of hours in the joint scheme compared to the sequential energy and reserve markets. This directly impacts the day-ahead decisions of the storage system in the sense that the storage system may lose profit in the day-ahead market at the benefit of gaining more opportunities in the reserve market. The storage system's profit in the joint energy and reserve markets is $6 \%$ higher that than in the sequential markets.

In Case 3, the storage system behaves strategically in the day-ahead market as joint energy and reserve settlement, while the balancing market is also considered to meet the net load deviations. We consider three foreseen scenarios representing the net load deviations uncertainty in the balancing stage, as given in the second column of Table $\mathrm{V}$. In the first scenario, the load deviation is zero, while in the second and third scenarios is $-5 \%$ and $+5 \%$ of demand, respectively. The corresponding probabilities are $0.90,0.05$ and 0.05 , respectively. The storage system's and generators' scheduling results are given in Tables $\mathrm{V}$ and $\mathrm{VI}$ respectively.

In Case 3, the storage system uses diverse offering and bidding strategies across hours to gain the maximum expected profit from different profit sources. In the charing hours, i.e., hours $1,3-5,8,10$ and 11-13, it bids at a high price $(\$ 400 / \mathrm{MWh})$ to be supplied, while the day-ahead price is set by cheap generator G1 to $\$ 12 / \mathrm{MWh}$. Unlike to energy bid price, the storage system offers its reserve capacity at

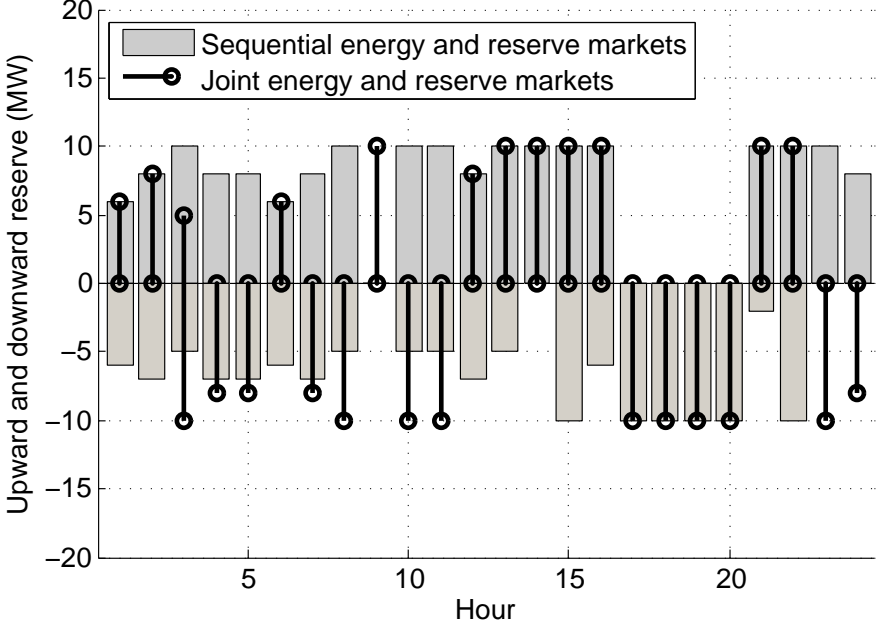

Fig. 2. Case 2: Hourly upward and downward reserve commitments of the storage system under joint and sequential energy and reserve market-clearing schemes

a low price $(\$ 5 / \mathrm{MWh})$ to be committed for providing the whole upward reserve and the half of downward reserve requirements of the market. This brings a market power for the storage system in the balancing stage, such that the balancing price under the third scenario (positive net load) increases to $\$ 400 / \mathrm{MWh}$. In the discharging hours, the storage system uses a different strategy. For example, the storage system offers its energy during the peak hours, i.e., hours 17 to 20 , at a low price $(\$ 12 / \mathrm{MWh})$ to make sure that it gets scheduled. This way, it is paid at the energy prices of peak hours, which are \$90/MWh and \$120/MWh. Note that with the same offer price of the storage system and generator, the bilevel optimization problem selects the storage systems' offer [23].

The storage system's profit in different three cases is compared in Fig. 3 It gains more expected profit in Case 3 
TABLE V

RESULTS FOR THE STORAGE SYSTEM IN CASE 3

\begin{tabular}{|c|c|c|c|c|c|c|c|c|c|c|c|c|}
\hline \multirow[b]{2}{*}{ Hr. } & \multirow[b]{2}{*}{$Q_{t, k}$} & \multicolumn{2}{|c|}{ Energy } & \multicolumn{4}{|c|}{ Reserve } & \multicolumn{2}{|c|}{ Balancing } & \multicolumn{3}{|c|}{ Market prices } \\
\hline & & $\begin{array}{c}p_{s, t}^{\text {dis }}, \\
p_{s, t}^{\text {ch }} \\
(\mathrm{MW})\end{array}$ & $\begin{array}{c}\widehat{O}_{s, t}^{\text {dis }}, \\
\widehat{o}_{s, t}^{\mathrm{ch}} \\
(\$ / \mathrm{MWh})\end{array}$ & $\begin{array}{c}r_{s, t}^{\mathrm{ch}, \mathrm{DN}}, \\
r_{s, t}^{\mathrm{ch}, \mathrm{UP}} \\
(\mathrm{MW})\end{array}$ & $\begin{array}{l}\widehat{o}_{s, t}^{\mathrm{ch}, \mathrm{DN}}, \\
\widehat{o}_{s, t}^{\mathrm{ch}, \mathrm{UP}} \\
(\$ / \mathrm{MWh})\end{array}$ & $\begin{array}{c}r_{s, t}^{\text {dis,DN }}, \\
r_{s, t}^{\text {dis, UP }} \\
\text { (MW) }\end{array}$ & $\begin{array}{c}\widehat{o}_{s, t}^{\text {dis,DN }}, \\
\widehat{o}_{s, t}^{\text {dis }, \mathrm{UP}} \\
(\$ / \mathrm{MWh})\end{array}$ & $\begin{array}{c}q_{s, t, k}^{\mathrm{ch}, \mathrm{DN}} \\
q_{s, t, k}^{\mathrm{ch}, \mathrm{UP}} \\
(\mathrm{MW})\end{array}$ & $\begin{array}{c}q_{s, t, k}^{\mathrm{dis}, \mathrm{DN}}, \\
q_{s, t, k}^{\mathrm{dis}, \mathrm{UP}} \\
\text { (MW) }\end{array}$ & $\begin{array}{c}\lambda_{t}^{\mathrm{EN}} \\
(\$ / \mathrm{MWh})\end{array}$ & $\begin{array}{c}\lambda_{t}^{\mathrm{DN}}, \\
\lambda_{t}^{\mathrm{UP}} \\
(\$ / \mathrm{MWh})\end{array}$ & $\begin{array}{r}\lambda_{t, 2}^{\mathrm{BL}}, \lambda_{t, 3}^{\mathrm{BI}} \\
(\$ / \mathrm{MWh})\end{array}$ \\
\hline 1 & $0,-3,3$ & -6 & 400 & $-3,6$ & 5,5 & & & $0,-3,3$ & & 12 & 5,5 & 12,400 \\
\hline 2 & $0,-4,4$ & & & & & 0,8 & 5 & & $0,0,4$ & 12 & 5,5 & 12,400 \\
\hline 3 & $0,-5,5$ & -10 & 400 & $-5,10$ & 5,5 & & & $0,-5,5$ & & 12 & 5,5 & 12,400 \\
\hline 4 & $0,-4,4$ & -8 & 400 & $-4,8$ & 5,5 & & & $0,-4,4$ & & 12 & 5,5 & 12,400 \\
\hline 5 & $0,-4,4$ & -8 & 400 & $-4,8$ & 5,5 & & & $0,-4,4$ & & 12 & 5,5 & 12,400 \\
\hline 6 & $0,-3,3$ & & & & & 0,6 & 5 & & $0,0,3$ & 12 & 5,5 & 12,400 \\
\hline 7 & $0,-4,4$ & & & & & 0,8 & 5 & & $0,0,4$ & 12 & 5,5 & 12,400 \\
\hline 8 & $0,-5,5$ & -10 & 400 & $-5,10$ & 5,5 & & & $0,-5,5$ & & 12 & 5,5 & 12,400 \\
\hline 9 & $0,-6,6$ & 2 & 12 & & & $-2,10$ & 5,15 & & $0,-2,4$ & 22 & 5,15 & 12,12 \\
\hline 10 & $0,-5,5$ & -10 & 400 & $-5,10$ & 5,5 & & & $0,-5,5$ & & 12 & 5,5 & 12,400 \\
\hline 11 & $0,-5,5$ & -10 & 400 & $-5,10$ & 5,5 & & & $0,-5,5$ & & 12 & 5,5 & 12,400 \\
\hline 12 & $0,-4,4$ & -8 & 400 & $-4,8$ & 5,5 & & & $0,-4,4$ & & 12 & 5,5 & 12,400 \\
\hline 13 & $0,-5,5$ & -10 & 400 & $-5,10$ & 5,5 & & & $0,-5,5$ & & 12 & 5,5 & 12,400 \\
\hline 14 & $0,-6,6$ & 2 & 12 & & & $-2,10$ & 5,15 & & $0,-2,4$ & 22 & 5,15 & 12,12 \\
\hline 15 & $0,-7,7$ & 10 & 30 & & & $-10,10$ & 5,15 & & $0,-7,3$ & 30 & 5,15 & 30,30 \\
\hline 16 & $0,-8,8$ & 6 & 30 & & & $-6,10$ & 5,25 & & $0,-6,2$ & 40 & 5,25 & 12,30 \\
\hline 17 & $0,-10,10$ & 10 & 12 & & & $-10,10$ & 5,40 & & $0,-10,10$ & 120 & 5,40 & 12,120 \\
\hline 18 & $0,-9,9$ & 12 & 12 & & & $-10,8$ & 5,25 & & $0,-9,8$ & 90 & 5,25 & 12,90 \\
\hline 19 & $0,-9,9$ & 12 & 12 & & & $-10,8$ & 5,25 & & $0,-9,8$ & 90 & 5,25 & 12,90 \\
\hline 20 & $0,-10,10$ & 10 & 12 & & & $-10,10$ & 5,40 & & $0,-10,10$ & 120 & 5,40 & 12,120 \\
\hline 21 & $0,-8,8$ & 6 & 30 & & & $-6,10$ & 5,25 & & $0,-6,2$ & 40 & 5,25 & 12,30 \\
\hline 22 & $0,-7,7$ & 10 & 30 & & & $-10,10$ & 5,15 & & $0,-7,3$ & 30 & 5,15 & 30,30 \\
\hline 23 & $0,-5,5$ & & & & & 0,10 & 5 & & $0,0,5$ & 12 & 5,5 & 12,400 \\
\hline 24 & $0,-4,4$ & & & & & 0,8 & 5 & & $0,0,4$ & 12 & 5,5 & 12,400 \\
\hline
\end{tabular}

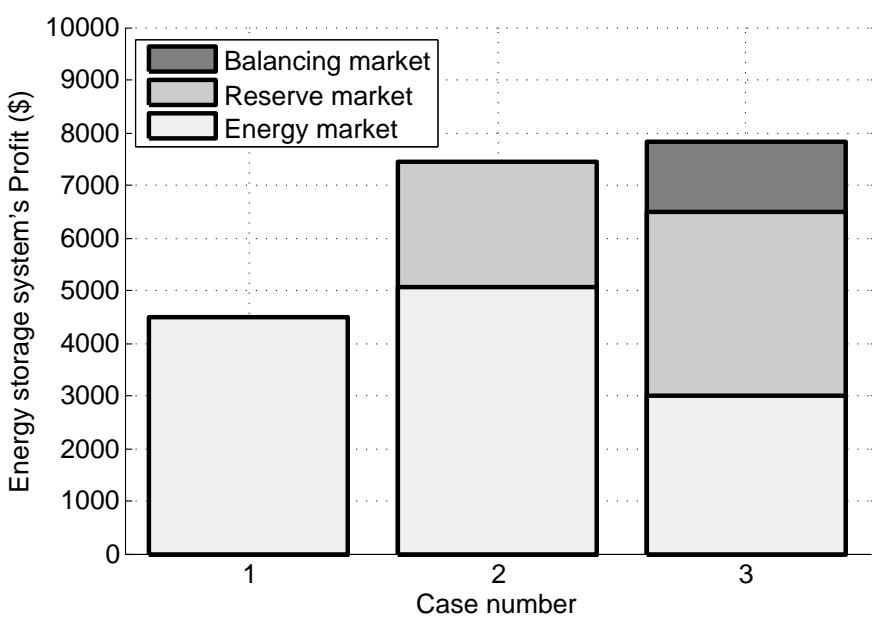

Fig. 3. The profit of the storage system in Cases 1, 2, and 3

compared to the first two cases due to availability of more business opportunities. In addition, the energy and reserve market prices for studied cases over 24 hours are illustrated in Fig. 4. Taking advantage of the price dependency in energy and reserve markets, the storage system's behaviour leads to higher energy prices in Case 2, compared to Case 1. This is why the storage system gains more profit in Case 2 compared to Case 1 in the day-ahead market, as evident in Fig. 3 In Case 3, compared to the first two cases, the storage system's actions lead to lower prices during charging hours and higher prices during discharging hours. Although the energy prices change to the storage system's advantage, the storage system's submitted quantities are lower in Case 3 compared to Case 2. That is why the storage system gains less in the day-ahead market in Case 3 compared to the first two cases (but more profit in total), as shown in Fig. 3 We now investigate how the expected profit of strategic storage system changes if the physical requirement constraints are enforced by scenario. Our simulation results show that such a profit in Case 3 reduces by $15 \%$ compared to that in a similar case when the physical constraints are enforced in expectation only.

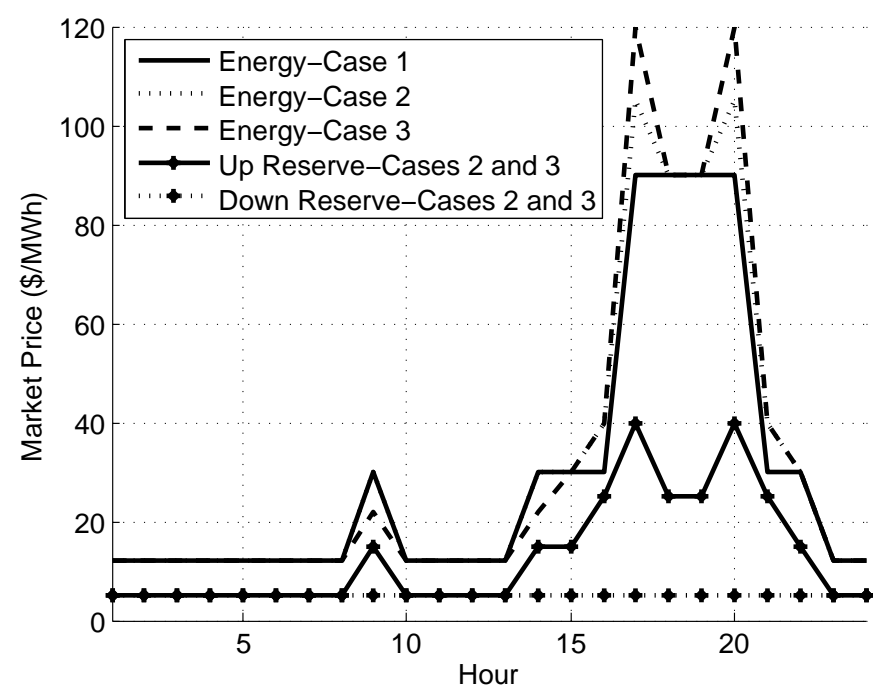

Fig. 4. Market prices in Cases 1, 2, and 3

Fig. 5 illustrates that the storage system's state of charge is comparatively smoother in Cases 2 and 3 compared to Case 1, since it participates in more hours. In Case 2 and 3, compared to Case 1 , the storage system participates in the energy market for a higher number of hours to be able to submit offers in the reserve market in those hours. In Case 3, compared to Case 2, the storage system submits lower offer quantities and higher offer prices. That is why the maximum storage system's state of charge is lower in Case 3 than that in Case 2. In all cases, the model was solved in less than two minutes on a standard desktop computer.

We also investigate how technical characteristics of storage system change its profitability. Fig. 6 shows the expected profit of the storage system as function of the capacity of charging and discharging devices. As expected, the storage system's profit depends on the capacity of both devices. Specifically, one device with a large capacity while the other one with a small capacity would not be a desirable configuration for the storage system in terms of its profitability. 


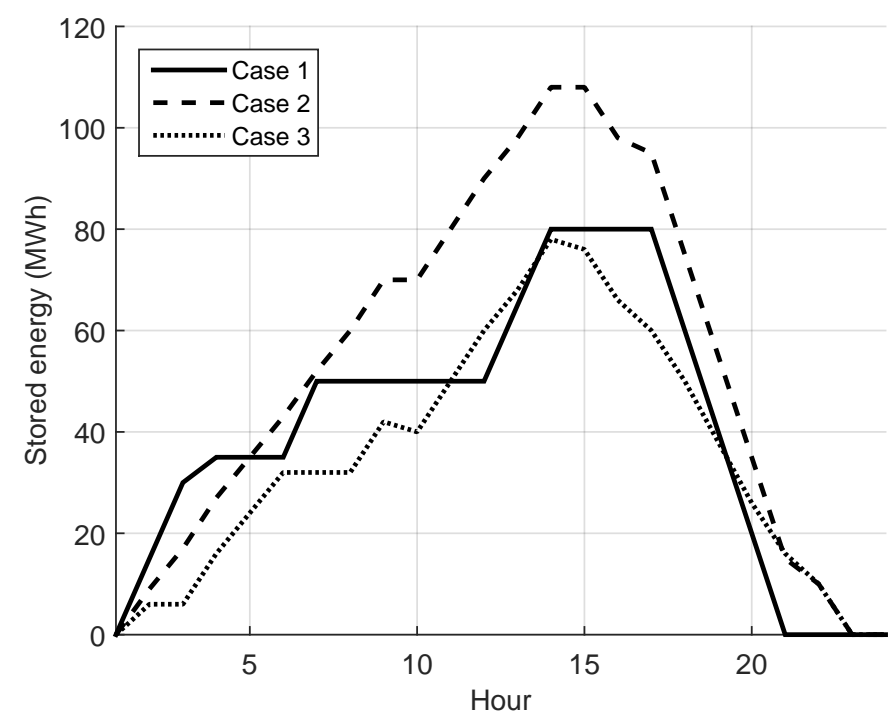

Fig. 5. The storage system's state of charge in Cases 1, 2, and 3

TABLE VI

RESULTS FOR GENERATORS IN CASE 3

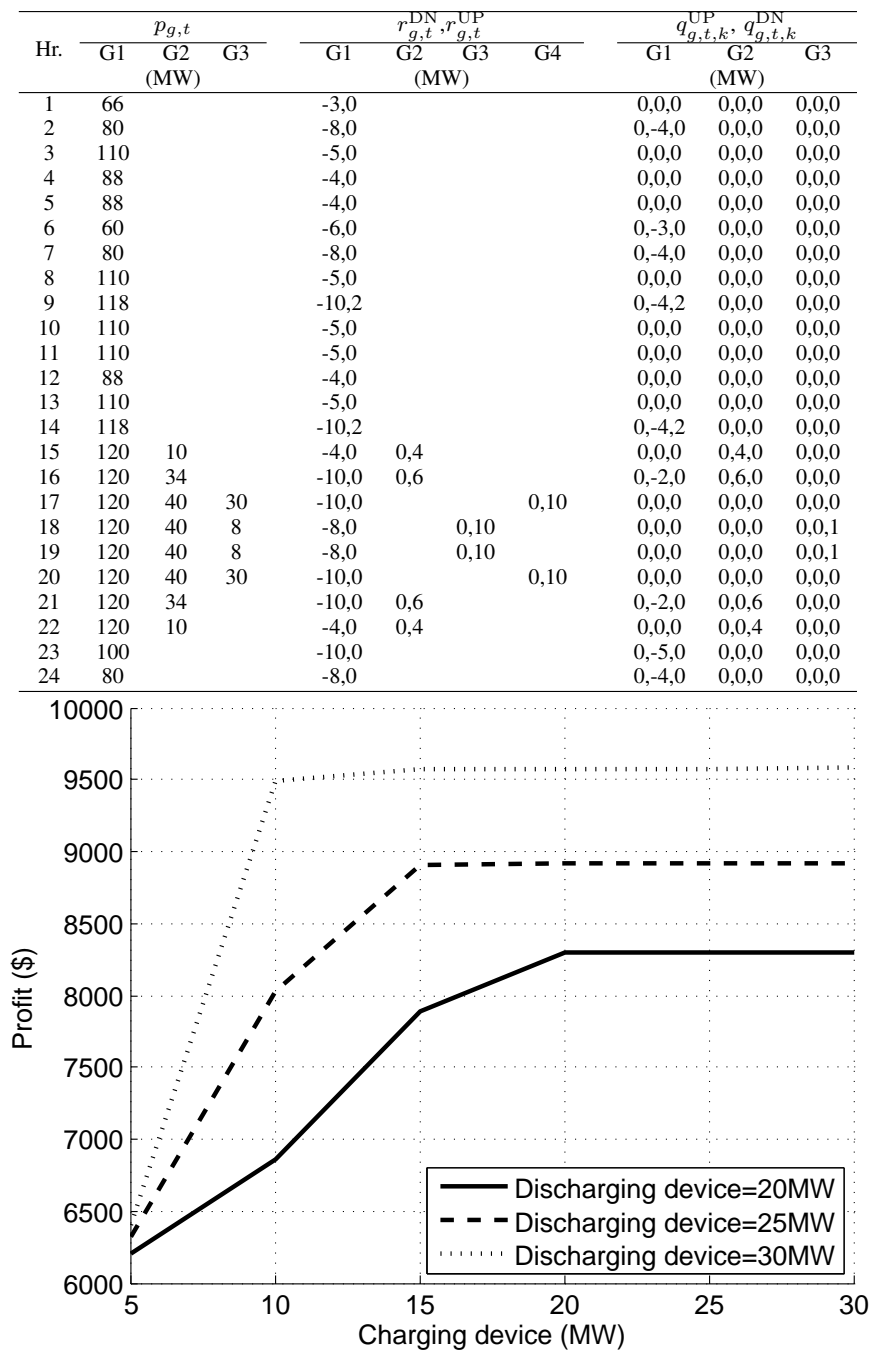

Fig. 6. The storage system's expected profit versus different capacity of charging and discharging devices
TABLE VII

DATA FOR GENERATORS (Alberta CASE)

\begin{tabular}{ccc}
\hline Unit No. & $\begin{array}{c}P_{g} \\
(\mathrm{MW})\end{array}$ & $\begin{array}{c}O_{g, t}^{\mathrm{EN}} \\
(\$ / \mathrm{MWh})\end{array}$ \\
\hline G1 & 5950 & 0 \\
G2 & 850 & 10 \\
G3 & 440 & 12 \\
G4 & 400 & 16 \\
G5 & 370 & 22 \\
G6 & 240 & 27 \\
G7 & 260 & 35 \\
G8 & 310 & 46 \\
G9 & 300 & 77 \\
G10 & 330 & 182 \\
G11 & 170 & 472 \\
G12 & 240 & 850 \\
G13 & 270 & 990 \\
G14 & 290 & 1000 \\
\hline & &
\end{tabular}

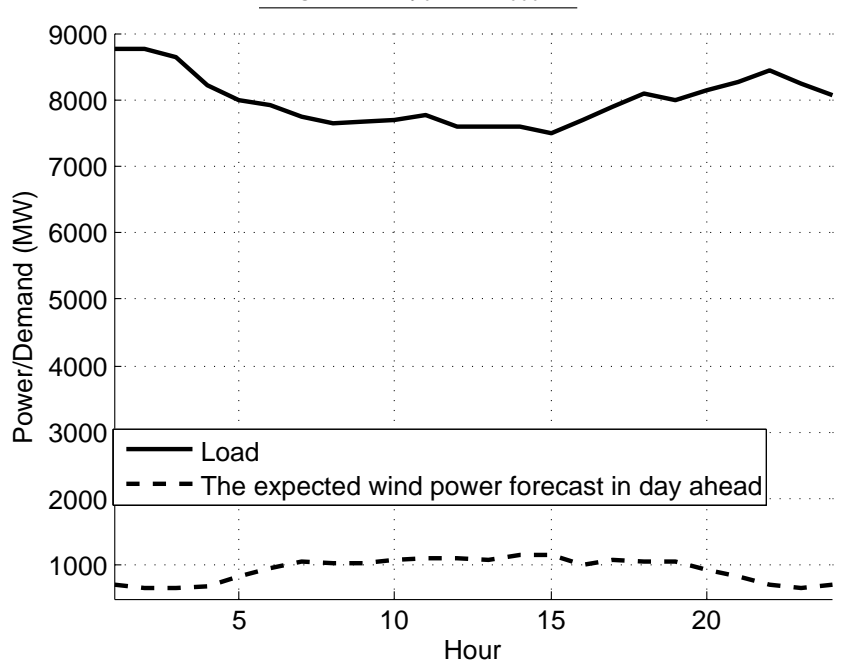

Fig. 7. The daily profile of load and the daily profile of aggregate wind power forecast in expectation

\section{B. A Case Study Based on Alberta Electricity Market}

Over the last few years, several energy storage projects including one large-scale compressed air energy storage were filed with the Alberta Electric System Operator (AESO) [24]. This motivates us to apply our proposed model to determine the optimal participation strategy of a large-scale compressed air energy storage system in the Alberta electricity market over an arbitrarily selected daily horizon (November 23, 2015). The technical characteristics of this storage system are given in Table II Note that this particular energy storage technology has different specifications for its charging and discharging devices.

More than 200 generation block offers at non-zero price were submitted to the market operator in the day considered. We model these generation blocks using 14 conventional generators, whose technical data are given in Table VII] Due to lack of some real-world information, we assume that each of 14 conventional generators offers for its reserve capability at a price equal to its half of energy offer price. In addition, we assume that the reserve capability of each conventional generator is equal to $20 \%$ of its installed capacity. Fig. 7 depicts the daily profile of load and the daily profile of aggregate wind production in expectation. The minimum reserve requirement of the market is considered $20 \%$ of expected day-ahead wind power. 


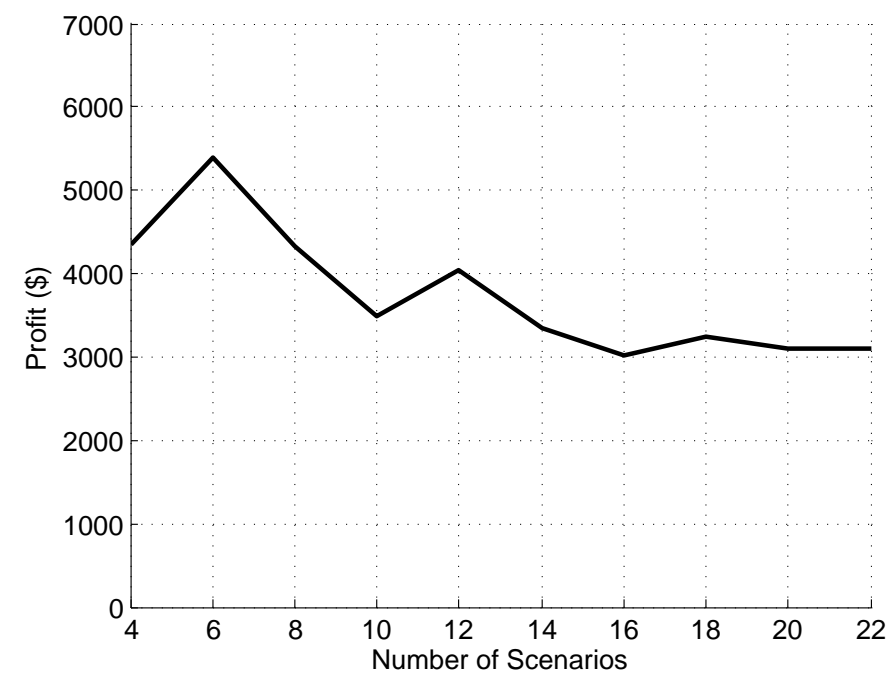

Fig. 8. The storage system's expected profit for different number of wind scenarios

In this paper, the uncertainty of net load deviation in realtime is incorporated into the model using a set of scenarios generated from the available forecast in the day-ahead stage. It is a straightforward extension to model additional sources of uncertainty, e.g., asset failure, but at the cost of increased computational burden. It is also possible to characterize load and wind uncertainties separately (instead of modeling net load uncertainty), which allows for considering their potential correlation. We generate 1000 daily wind scenarios considering a normal distribution with the standard deviation of $10 \%$. A backward scenario reduction method [25] is then used to select the most representative scenarios. We first start with four representative scenarios and then increase the number of scenarios. This way, we derive the storage system's expected profit achieved by our proposed model for different number of representative scenarios as illustrated in Fig. 8, Observe that such a profit reaches to a stability in a case for which the number of scenarios is 16. A higher number of scenarios does not considerably change the storage system's profit. This implicitly means this number of scenarios is representative enough.

We now investigate the computational time for solving the model. One important observation, as illustrated in Fig. 9 for a case with 15 net load scenarios, is that the MW-step (horizontal axis) used within the binary expansion method (Appendix B) considerably affects the computational time while roughly changes the expected profit of the storage system. The smaller MW-step (e.g., $25 \mathrm{MW}$ ) provides a tighter feasible space, and thus a higher accuracy for binary expansion approximation, but at the cost of significant computational time. The bigger MWstep reduces the accuracy of approximation method and also lowers the computational time. Note that an iterative approach [26] can be used, so that the feasible space of discretized variable can be tightened based on the optimal value achieved for that variable in the previous iteration.

\section{CONCLUSION}

In this paper, we demonstrate that the multiple uses of a storage system can bring substantive value to it. The pool strategy of a storage system as a price-maker facility in the energy, reserve, and balancing markets is proposed and formulated. The uncertainty in the balancing market is reflected through a set of scenarios. To solve this problem, it is recast to an MPEC and then linearized. The resulting problem is an MILP. The storage system's bidding strategy is discussed in the energy-only market, the joint energy and reserve markets, and the joint energy and reserve markets considering the balancing markets. The results show that the storage system's strategic decisions in multiple markets significantly change compared to a single market. It is also explained how storage strategic decisions in multiple markets are dependent on each other.

\section{APPENDIX A: KKT CONDITIONS OF LOWER-LEVEL PROBLEMS}

This appendix includes the KKT conditions of lower-level problems (b) and (c).

The KKT conditions associated with lower-level problem (b.1)-(b.22) are given by d.1 - d.42 below:

$$
\begin{aligned}
& \widehat{o}_{s, t}^{\mathrm{dis}}-\lambda_{t}^{\mathrm{EN}}+\bar{\mu}_{s, t}^{\mathrm{dis}}-\underline{\mu}_{s, t}^{\mathrm{dis}}-\mu_{s, t}^{\mathrm{dis}, \mathrm{DN}}=0 \quad \forall s, \forall t \\
& -\widehat{o}_{s, t}^{\mathrm{ch}}+\lambda_{t}^{\mathrm{EN}}+\bar{\mu}_{s, t}^{\mathrm{ch}}-\underline{\mu}_{s, t}^{\mathrm{ch}}-\mu_{s, t}^{\mathrm{ch}, \mathrm{UP}}=0 \quad \forall s, \forall t \\
& O_{g, t}^{\mathrm{EN}}-\lambda_{t}^{\mathrm{EN}}+\bar{\mu}_{g, t}-\underline{\mu}_{g, t}+\mu_{g, t}^{\mathrm{UP}}-\mu_{g, t}^{\mathrm{DN}}=0 \\
& \forall g, \forall t \\
& -U_{d, t}^{\mathrm{EN}}+\lambda_{t}^{\mathrm{EN}}+\bar{\mu}_{d, t}-\underline{\mu}_{d, t}+\mu_{d, t}^{\mathrm{UP}}-\mu_{d, t}^{\mathrm{DN}}=0 \\
& \forall d, \forall t \\
& \widehat{o}_{s, t}^{\mathrm{dis}, \mathrm{UP}}-\lambda_{t}^{\mathrm{UP}}+\bar{\mu}_{s, t}^{\mathrm{dis}, \mathrm{UP}}-\underline{\mu}_{s, t}^{\mathrm{dis}, \mathrm{UP}}=0 \quad \forall s, \forall t \\
& \widehat{o}_{s, t}^{\mathrm{dis}, \mathrm{DN}}-\lambda_{t}^{\mathrm{DN}}+\bar{\mu}_{s, t}^{\mathrm{dis}, \mathrm{DN}}-\underline{\mu}_{s, t}^{\mathrm{dis}, \mathrm{DN}}+\mu_{s, t}^{\mathrm{dis}, \mathrm{DN}}=0 \\
& \forall s, \forall t \\
& \widehat{o}_{s, t}^{\mathrm{ch}, \mathrm{UP}}-\lambda_{t}^{\mathrm{UP}}+\bar{\mu}_{s, t}^{\mathrm{ch}, \mathrm{UP}}-\underline{\mu}_{s, t}^{\mathrm{ch}, \mathrm{UP}}+\mu_{s, t}^{\mathrm{ch}, \mathrm{UP}}=0 \\
& \forall s, \forall t \\
& \widehat{o}_{s, t}^{\mathrm{ch}, \mathrm{DN}}-\lambda_{t}^{\mathrm{DN}}+\bar{\mu}_{s, t}^{\mathrm{ch}, \mathrm{DN}}-\underline{\mu}_{s, t}^{\mathrm{ch}, \mathrm{DN}}=0 \quad \forall s, \forall t \\
& O_{g, t}^{\mathrm{RS}}-\lambda_{t}^{\mathrm{UP}}+\bar{\mu}_{g, t}^{\mathrm{UP}}-\underline{\mu}_{g, t}^{\mathrm{UP}}+\mu_{g, t}^{\mathrm{UP}}=0 \quad \forall g, \forall t \\
& O_{g, t}^{\mathrm{RS}}-\lambda_{t}^{\mathrm{DN}}+\bar{\mu}_{g, t}^{\mathrm{DN}}-\underline{\mu}_{g, t}^{\mathrm{DN}}+\mu_{g, t}^{\mathrm{DN}}=0 \quad \forall g, \forall t \\
& U_{d, t}^{\mathrm{RS}}-\lambda_{t}^{\mathrm{UP}}+\bar{\mu}_{d, t}^{\mathrm{UP}}-\underline{\mu}_{d, t}^{\mathrm{UP}}+\mu_{d, t}^{\mathrm{UP}}=0 \quad \forall d, \forall t \\
& U_{d, t}^{\mathrm{RS}}-\lambda_{t}^{\mathrm{DN}}+\bar{\mu}_{d, t}^{\mathrm{DN}}-\underline{\mu}_{d, t}^{\mathrm{DN}}+\mu_{d, t}^{\mathrm{DN}}=0 \quad \forall d, \forall t \\
& 0 \leq p_{g, t} \perp \underline{\mu}_{g, t} \geq 0 \quad \forall g, \forall t \\
& 0 \leq\left(P_{g}-p_{g, t}\right) \perp \bar{\mu}_{g, t} \geq 0 \quad \forall g, \forall t \\
& 0 \leq p_{d, t} \underline{\mu}_{d, t} \geq 0 \quad \forall d, \forall t \\
& 0 \leq\left(P_{d, t}-p_{d, t}\right) \perp \bar{\mu}_{d, t} \geq 0 \quad \forall d, \forall t \\
& 0 \leq p_{s, t}^{\mathrm{ch}} \perp \underline{\mu}_{s, t}^{\mathrm{ch}} \geq 0 \quad \forall s, \forall t \\
& 0 \leq\left(\widehat{p}_{s, t}^{\mathrm{ch}}-p_{s, t}^{\mathrm{ch}}\right) \perp \bar{\mu}_{s, t}^{\mathrm{ch}} \geq 0 \quad \forall s, \forall t
\end{aligned}
$$




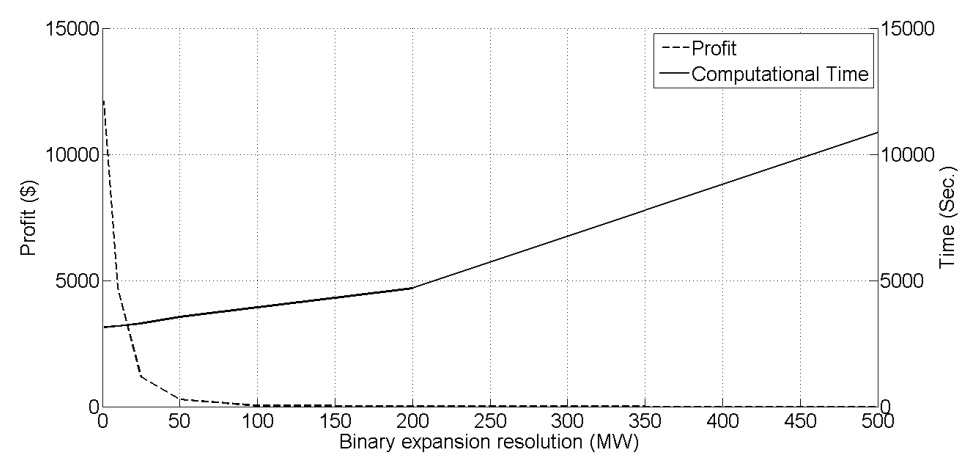

Fig. 9. The expected profit of the storage system and computational time for solving the proposed model as a function of MW-step used within the binary expansion method

$$
\begin{aligned}
& 0 \leq p_{s, t}^{\text {dis }} \perp \underline{\mu}_{s, t}^{\text {dis }} \geq 0 \quad \forall s, \forall t \\
& 0 \leq\left(\widehat{p}_{s, t}^{\text {dis }}-p_{s, t}^{\text {dis }}\right) \perp \bar{\mu}_{s, t}^{\text {dis }} \geq 0 \quad \forall s, \forall t \\
& 0 \leq r_{s, t}^{\mathrm{ch}, \mathrm{UP}} \perp \underline{\mu}_{s, t}^{\mathrm{ch}, \mathrm{UP}} \geq 0 \quad \forall s, \forall t \\
& 0 \leq\left(\widehat{r}_{s, t}^{\mathrm{ch}, \mathrm{UP}}-r_{s, t}^{\mathrm{ch}, \mathrm{UP}}\right) \perp \bar{\mu}_{s, t}^{\mathrm{ch}, \mathrm{UP}} \geq 0 \quad \forall s, \forall t \\
& 0 \leq r_{s, t}^{\mathrm{ch}, \mathrm{DN}} \perp \underline{\mu}_{s, t}^{\mathrm{ch}, \mathrm{DN}} \geq 0 \quad \forall s, \forall t \\
& 0 \leq\left(\widehat{r}_{s, t}^{\mathrm{ch}, \mathrm{DN}}-r_{s, t}^{\mathrm{ch}, \mathrm{DN}}\right) \perp \bar{\mu}_{s, t}^{\mathrm{ch}, \mathrm{DN}} \geq 0 \quad \forall s, \forall t \\
& 0 \leq r_{s, t}^{\mathrm{dis}, \mathrm{UP}} \perp \underline{\mu}_{s, t}^{\mathrm{dis}, \mathrm{UP}} \geq 0 \quad \forall s, \forall t \\
& 0 \leq\left(\widehat{r}_{s, t}^{\mathrm{dis}, \mathrm{UP}}-r_{s, t}^{\mathrm{dis}, \mathrm{UP}}\right) \perp \bar{\mu}_{s, t}^{\mathrm{dis}, \mathrm{UP}} \geq 0 \quad \forall s, \forall t \\
& 0 \leq r_{s, t}^{\mathrm{dis}, \mathrm{DN}} \perp \underline{\mu}_{s, t}^{\mathrm{dis}, \mathrm{DN}} \geq 0 \quad \forall s, \forall t \\
& 0 \leq\left(\widehat{r}_{s, t}^{\mathrm{dis}, \mathrm{DN}}-r_{s, t}^{\mathrm{dis}, \mathrm{DN}}\right) \perp \bar{\mu}_{s, t}^{\mathrm{dis}, \mathrm{DN}} \geq 0 \quad \forall s, \forall t \\
& 0 \leq\left(p_{s, t}^{\mathrm{ch}}-r_{s, t}^{\mathrm{ch}, \mathrm{UP}}\right) \perp \mu_{s, t}^{\mathrm{ch}, \mathrm{UP}} \geq 0 \quad \forall s, \forall t \\
& 0 \leq\left(p_{s, t}^{\mathrm{dis}}-r_{s, t}^{\mathrm{dis}, \mathrm{DN}}\right) \perp \mu_{s, t}^{\mathrm{dis}, \mathrm{DN}} \geq 0 \quad \forall s, \forall t \\
& 0 \leq r_{g, t}^{\mathrm{UP}} \perp \underline{\mu}_{g, t}^{\mathrm{UP}} \geq 0 \quad \forall g, \forall t \\
& 0 \leq\left(R_{g}^{\mathrm{UP}}-r_{g, t}^{\mathrm{UP}}\right) \perp \bar{\mu}_{g, t}^{\mathrm{UP}} \geq 0 \quad \forall g, \forall t \\
& 0 \leq r_{g, t}^{\mathrm{DN}} \perp \underline{\mu}_{g, t}^{\mathrm{DN}} \geq 0 \quad \forall g, \forall t \\
& 0 \leq\left(R_{g}^{\mathrm{DN}}-r_{g, t}^{\mathrm{DN}}\right) \perp \bar{\mu}_{g, t}^{\mathrm{DN}} \geq 0 \quad \forall g, \forall t \\
& 0 \leq\left(P_{g}-p_{g, t}-r_{g, t}^{\mathrm{UP}}\right) \perp \mu_{g, t}^{\mathrm{UP}} \geq 0 \quad \forall g, \forall t \\
& 0 \leq\left(p_{g, t}-r_{g, t}^{\mathrm{DN}}\right) \perp \mu_{g, t}^{\mathrm{DN}} \geq 0 \quad \forall g, \forall t \\
& 0 \leq r_{d, t}^{\mathrm{UP}} \perp \underline{\mu}_{d, t}^{\mathrm{UP}} \geq 0 \quad \forall d, \forall t \\
& 0 \leq\left(R_{d, t}^{\mathrm{UP}}-r_{d, t}^{\mathrm{UP}}\right) \perp \bar{\mu}_{d, t}^{\mathrm{UP}} \geq 0 \quad \forall d, \forall t \\
& 0 \leq r_{d, t}^{\mathrm{DN}} \perp_{\mu_{d, t}^{\mathrm{DN}}} \geq 0 \quad \forall d, \forall t \\
& 0 \leq\left(R_{d, t}^{\mathrm{DN}}-r_{d, t}^{\mathrm{DN}}\right) \perp \bar{\mu}_{d, t}^{\mathrm{DN}} \geq 0 \quad \forall d, \forall t \\
& 0 \leq\left(P_{d, t}-p_{d, t}-r_{d, t}^{\mathrm{DN}}\right) \perp \mu_{d, t}^{\mathrm{DN}} \geq 0 \quad \forall d, \forall t \\
& 0 \leq\left(p_{d, t}-r_{d, t}^{\mathrm{UP}}\right) \perp \mu_{d, t}^{\mathrm{UP}} \geq 0 \quad \forall d, \forall t
\end{aligned}
$$

Likewise, the KKT conditions associated with lower-level problems (c.1)-(c.11), one per scenario, are given by e.11(e.27) below:

$$
\widehat{o}_{s, t}^{\mathrm{dis}}-\lambda_{t, k}^{\mathrm{BL}}+\bar{\rho}_{s, t, k}^{\mathrm{dis}, \mathrm{UP}}-\underline{\rho}_{s, t, k}^{\mathrm{dis}, \mathrm{UP}}=0 \quad \forall s, \forall t, \forall k
$$

$$
\begin{aligned}
& -\widehat{o}_{s, t}^{\mathrm{dis}}+\lambda_{t, k}^{\mathrm{BL}}+\bar{\rho}_{s, t, k}^{\mathrm{dis}, \mathrm{DN}}-\underline{\rho}_{s, t, k}^{\mathrm{dis}, \mathrm{DN}}=0 \quad \forall s, \forall t, \forall k \\
& \widehat{o}_{s, t}^{\mathrm{ch}}-\lambda_{t, k}^{\mathrm{BL}}+\bar{\rho}_{s, t, k}^{\mathrm{ch}, \mathrm{UP}}-\underline{\rho}_{s, t, k}^{\mathrm{ch}, \mathrm{UP}}=0 \quad \forall s, \forall t, \forall k \\
& -\widehat{o}_{s, t}^{\mathrm{ch}}+\lambda_{t, k}^{\mathrm{BL}}+\bar{\rho}_{s, t, k}^{\mathrm{ch}, \mathrm{DN}}-\underline{\rho}_{s, t, k}^{\mathrm{ch}, \mathrm{DN}}=0 \quad \forall s, \forall t, \forall k \\
& O_{g, t}^{\mathrm{EN}}-\lambda_{t, k}^{\mathrm{BL}}+\bar{\rho}_{g, t, k}^{\mathrm{UP}}-\underline{\rho}_{g, t, k}^{\mathrm{UP}}=0 \quad \forall g, \forall t, \forall k \\
& -O_{g, t}^{\mathrm{EN}}+\lambda_{t, k}^{\mathrm{BL}}+\bar{\rho}_{g, t, k}^{\mathrm{DN}}-\underline{\rho}_{g, t, k}^{\mathrm{DN}}=0 \quad \forall g, \forall t, \forall k \\
& U_{d, t}^{\mathrm{EN}}-\lambda_{t, k}^{\mathrm{BL}}+\bar{\rho}_{d, t, k}^{\mathrm{UP}}-\underline{\rho}_{d, t, k}^{\mathrm{UP}}=0 \quad \forall d, \forall t, \forall k \\
& -U_{d, t}^{\mathrm{EN}}+\lambda_{t, k}^{\mathrm{BL}}+\bar{\rho}_{d, t, k}^{\mathrm{DN}}-\underline{\rho}_{d, t, k}^{\mathrm{DN}}=0 \quad \forall d, \forall t, \forall k \\
& V_{d, t}-\lambda_{t, k}^{\mathrm{BL}}+\bar{\rho}_{d, t, k}-\underline{\rho}_{d, t, k}=0 \quad \forall d, \forall t, \forall k \\
& 0 \leq q_{g, t, k}^{\mathrm{UP}} \perp \underline{\rho}_{g, t, k}^{\mathrm{UP}} \geq 0 \quad \forall g, \forall t, \forall k \\
& 0 \leq\left(r_{g, t}^{\mathrm{UP}}-q_{g, t, k}^{\mathrm{UP}}\right) \perp \bar{\rho}_{g, t, k}^{\mathrm{UP}} \geq 0 \quad \forall g, \forall t, \forall k \\
& 0 \leq q_{g, t, k}^{\mathrm{DN}} \perp \underline{\rho}_{g, t, k}^{\mathrm{DN}} \geq 0 \quad \forall g, \forall t, \forall k \\
& 0 \leq\left(r_{g, t}^{\mathrm{DN}}-q_{g, t, k}^{\mathrm{DN}}\right) \perp \bar{\rho}_{g, t, k}^{\mathrm{DN}} \geq 0 \quad \forall g, \forall t, \forall k \\
& 0 \leq q_{d, t, k}^{\mathrm{UP}} \perp \underline{\rho}_{d, t, k}^{\mathrm{UP}} \geq 0 \quad \forall d, \forall t, \forall k \\
& 0 \leq\left(r_{g, t}^{\mathrm{UP}}-q_{d, t, k}^{\mathrm{UP}}\right) \perp \bar{\rho}_{d, t, k}^{\mathrm{UP}} \geq 0 \quad \forall d, \forall t, \forall k \\
& 0 \leq q_{d, t, k}^{\mathrm{DN}} \perp \underline{\rho}_{d, t, k}^{\mathrm{DN}} \geq 0 \quad \forall d, \forall t, \forall k \\
& 0 \leq\left(r_{d, t}^{\mathrm{DN}}-q_{d, t, k}^{\mathrm{DN}}\right) \perp \bar{\rho}_{d, t, k}^{\mathrm{DN}} \geq 0 \quad \forall d, \forall t, \forall k \\
& 0 \leq q_{s, t, k}^{\mathrm{dis}, \mathrm{UP}} \perp \underline{\rho}_{s, t, k}^{\mathrm{dis}, \mathrm{UP}} \geq 0 \quad \forall s, \forall t, \forall k \\
& 0 \leq\left(r_{s, t}^{\mathrm{dis}, \mathrm{UP}}-q_{s, t, k}^{\mathrm{dis}, \mathrm{UP}}\right) \perp \bar{\rho}_{s, t, k}^{\mathrm{dis}, \mathrm{UP}} \geq 0 \quad \forall s, \forall t, \forall k \\
& 0 \leq q_{s, t, k}^{\mathrm{dis}, \mathrm{DN}} \perp \underline{\rho}_{s, t, k}^{\mathrm{dis}, \mathrm{DN}} \geq 0 \quad \forall s, \forall t, \forall k \\
& 0 \leq\left(r_{s, t}^{\mathrm{dis}, \mathrm{DN}}-q_{s, t, k}^{\mathrm{dis}, \mathrm{DN}}\right) \perp \bar{\rho}_{s, t, k}^{\mathrm{dis}, \mathrm{DN}} \geq 0 \quad \forall s, \forall t, \forall k \\
& 0 \leq q_{s, t, k}^{\mathrm{ch}, \mathrm{UP}} \perp \underline{\rho}_{s, t, k}^{\mathrm{ch}, \mathrm{UP}} \geq 0 \quad \forall s, \forall t, \forall k \\
& 0 \leq\left(r_{s, t}^{\mathrm{ch}, \mathrm{UP}}-q_{s, t, k}^{\mathrm{ch}, \mathrm{UP}}\right) \perp \bar{\rho}_{s, t, k}^{\mathrm{ch}, \mathrm{UP}} \geq 0 \quad \forall s, \forall t, \forall k \\
& 0 \leq q_{s, t, k}^{\mathrm{ch}, \mathrm{DN}} \perp \underline{\rho}_{s, t, k}^{\mathrm{ch}, \mathrm{DN}} \geq 0 \quad \forall s, \forall t, \forall k \\
& 0 \leq\left(r_{s, t}^{\mathrm{ch}, \mathrm{DN}}-q_{s, t, k}^{\mathrm{ch}, \mathrm{DN}}\right) \perp \bar{\rho}_{s, t, k}^{\mathrm{ch}, \mathrm{DN}} \geq 0 \quad \forall s, \forall t, \forall k \\
& 0 \leq l_{d, t, k} \perp_{d, t, k} \geq 0 \quad \forall d, \forall t, \forall k \\
& 0 \leq\left(p_{d, t}-l_{d, t, k}\right) \perp \bar{\rho}_{d, t, k} \geq 0 \quad \forall d, \forall t, \forall k
\end{aligned}
$$




\section{APPENDIX B: LINEARIZATION OF BILINEAR TERMS IN OBJECTIVE FUNCTION a.1)}

This appendix explains the linearization process of bilinear terms within the upper-level objective function a.1. First of all, we derive the strong duality equality (f.1) below. This equality corresponds to lower-level problem (b), and enforces that the value of its primal objective function, i.e., (b.1), is equal to that of its dual problem at the optimal solution:

$$
\begin{aligned}
& -\sum_{s=1}^{N_{s}}\left(\widehat{o}_{s, t}^{\mathrm{ch}} \cdot p_{s, t}^{\mathrm{ch}}-\widehat{o}_{s, t}^{\mathrm{dis}} \cdot p_{s, t}^{\mathrm{dis}}\right)-\sum_{d=1}^{N_{d}} U_{d, t}^{\mathrm{EN}} \cdot p_{d, t} \\
& \quad+\sum_{g=1}^{N_{g}} O_{g, t}^{\mathrm{EN}} \cdot p_{g, t}+\sum_{s=1}^{N_{s}}\left(\widehat{o}_{s, t}^{\mathrm{ch}, \mathrm{UP}} \cdot r_{s, t}^{\mathrm{ch}, \mathrm{UP}}+\widehat{o}_{s, t}^{\mathrm{ch}, \mathrm{DN}} \cdot r_{s, t}^{\mathrm{ch}, \mathrm{DN}}\right. \\
& \left.+\widehat{o}_{s, t}^{\mathrm{dis}, \mathrm{UP}} \cdot r_{s, t}^{\mathrm{dis}, \mathrm{UP}}+\widehat{o}_{s, t}^{\mathrm{dis}, \mathrm{DN}} \cdot r_{s, t}^{\mathrm{dis}, \mathrm{DN}}\right) \\
& \quad+\sum_{g=1}^{N_{g}} O_{g, t}^{\mathrm{RS}} \cdot\left(r_{g, t}^{\mathrm{UP}}+r_{g, t}^{\mathrm{DN}}\right)+\sum_{d=1}^{N_{d}} U_{d, t}^{\mathrm{RS}} \cdot\left(r_{d, t}^{\mathrm{UP}}+r_{d, t}^{\mathrm{DN}}\right) \\
& =-\sum_{s=1}^{N_{s}}\left(\bar{\mu}_{s, t}^{\mathrm{ch}} \cdot \widehat{p}_{s, t}^{\mathrm{ch}}+\bar{\mu}_{s, t}^{\mathrm{dis}} \cdot \widehat{p}_{s, t}^{\mathrm{dis}}\right. \\
& +\bar{\mu}_{s, t}^{\mathrm{ch}, \mathrm{UP}} \cdot \widehat{r}_{s, t}^{\mathrm{ch}, \mathrm{UP}}+\bar{\mu}_{s, t}^{\mathrm{ch}, \mathrm{DN}} \cdot \widehat{r}_{s, t}^{\mathrm{ch}, \mathrm{DN}}+\bar{\mu}_{s, t}^{\mathrm{dis}, \mathrm{UP}} \cdot \widehat{r}_{s, t}^{\mathrm{dis}, \mathrm{UP}} \\
& \left.+\bar{\mu}_{s, t}^{\mathrm{dis}, \mathrm{DN}} \cdot \widehat{r}_{s, t}^{\mathrm{dis}, \mathrm{DN}}\right)+X_{t}^{\mathrm{DA}} \forall t
\end{aligned}
$$

where,

$$
\begin{aligned}
& X_{t}^{\mathrm{DA}}=-\sum_{d=1}^{N_{d}} \bar{\mu}_{d, t} \cdot P_{d, t}-\sum_{g=1}^{N_{g}} \bar{\mu}_{g, t} \cdot P_{g} \\
& -\sum_{d=1}^{N_{d}} \bar{\mu}_{d, t}^{\mathrm{UP}} \cdot R_{d, t}^{\mathrm{UP}}-\sum_{d=1}^{N_{d}} \bar{\mu}_{d, t}^{\mathrm{DN}} \cdot R_{d, t}^{\mathrm{DN}} \\
& -\sum_{g=1}^{N_{g}} \bar{\mu}_{g, t}^{\mathrm{UP}} \cdot R_{g}^{\mathrm{UP}}-\sum_{g=1}^{N_{g}} \bar{\mu}_{g, t}^{\mathrm{DN}} \cdot R_{g}^{\mathrm{DN}} \\
& -\sum_{d=1}^{N_{d}} \mu_{d, t}^{\mathrm{DN}} \cdot P_{d, t}-\sum_{g=1}^{N_{g}} \mu_{g, t}^{\mathrm{UP}} \cdot P_{g} \\
& +\left(\lambda_{t}^{\mathrm{UP}} \cdot R_{t}^{\mathrm{UP}}+\lambda_{t}^{\mathrm{DN}} \cdot R_{t}^{\mathrm{DN}}\right) \forall t
\end{aligned}
$$

From d.18, ,d.20, d.22), d.24, (d.26, and d.28):

$$
\begin{aligned}
& \bar{\mu}_{s, t}^{\mathrm{ch}} \cdot \widehat{p}_{s, t}^{\mathrm{ch}}=\bar{\mu}_{s, t}^{\mathrm{ch}} \cdot p_{s, t}^{\mathrm{ch}} \forall s, \forall t \\
& \bar{\mu}_{s, t}^{\mathrm{dis}} \cdot \widehat{p}_{s, t}^{\mathrm{dis}}=\bar{\mu}_{s, t}^{\mathrm{dis}} \cdot p_{s, t}^{\mathrm{dis}} \forall s, \forall t \\
& \bar{\mu}_{s, t}^{\mathrm{ch}, \mathrm{UP}} \cdot \widehat{r}_{s, t}^{\mathrm{ch}, \mathrm{UP}}=\bar{\mu}_{s, t}^{\mathrm{ch}, \mathrm{UP}} \cdot r_{s, t}^{\mathrm{ch}, \mathrm{UP}} \forall s, \forall t \\
& \bar{\mu}_{s, t}^{\mathrm{ch}, \mathrm{DN}} \cdot \widehat{r}_{s, t}^{\mathrm{ch}, \mathrm{DN}}=\bar{\mu}_{s, t}^{\mathrm{ch}, \mathrm{DN}} \cdot r_{s, t}^{\mathrm{ch}, \mathrm{DN}} \forall s, \forall t \\
& \bar{\mu}_{s, t}^{\mathrm{dis}, \mathrm{UP}} \cdot \widehat{r}_{s, t}^{\mathrm{dis}, \mathrm{UP}}=\bar{\mu}_{s, t}^{\mathrm{dis}, \mathrm{UP}} \cdot r_{s, t}^{\mathrm{dis}, \mathrm{UP}} \quad \forall s, \forall t \\
& \bar{\mu}_{s, t}^{\mathrm{dis}, \mathrm{DN}} . r_{s, t}^{\mathrm{dis}, \mathrm{DN}}=\bar{\mu}_{s, t}^{\mathrm{dis}, \mathrm{DN}} . r_{s, t}^{\mathrm{dis}, \mathrm{DN}} \quad \forall s, \forall t
\end{aligned}
$$

Substituting (f.3)-f.8) in f.1 yields

$$
\sum_{s=1}^{N_{s}}\left(\left[-\widehat{o}_{s, t}^{\mathrm{ch}}+\bar{\mu}_{s, t}^{\mathrm{ch}}\right] \cdot p_{s, t}^{\mathrm{ch}}+\left[\widehat{o}_{s, t}^{\mathrm{dis}}+\bar{\mu}_{s, t}^{\mathrm{dis}}\right] \cdot p_{s, t}^{\mathrm{dis}}\right.
$$

$$
\begin{aligned}
& +\left[\widehat{o}_{s, t}^{\mathrm{ch}, \mathrm{UP}}+\bar{\mu}_{s, t}^{\mathrm{ch}, \mathrm{UP}}\right] \cdot r_{s, t}^{\mathrm{ch}, \mathrm{UP}} \\
& +\left[\widehat{o}_{s, t}^{\mathrm{ch}, \mathrm{DN}}+\bar{\mu}_{s, t}^{\mathrm{dis}, \mathrm{DN}}\right] \cdot r_{s, t}^{\mathrm{ch}, \mathrm{DN}}+\left[\widehat{o}_{s, t}^{\mathrm{dis}, \mathrm{UP}}+\bar{\mu}_{s, t}^{\mathrm{dis}, \mathrm{UP}}\right] \cdot r_{s, t}^{\mathrm{dis}, \mathrm{UP}} \\
& \left.+\left[\widehat{o}_{s, t}^{\mathrm{dis}, \mathrm{DN}}+\bar{\mu}_{s, t}^{\mathrm{dis}, \mathrm{UP}}\right] \cdot r_{s, t}^{\mathrm{dis}, \mathrm{DN}}\right) \\
& =\sum_{d=1}^{N_{d}} U_{d, t}^{\mathrm{EN}} \cdot p_{d, t}-\sum_{g=1}^{N_{g}} O_{g, t}^{\mathrm{EN}} \cdot p_{g, t} \\
& -\sum_{g=1}^{N_{g}} O_{g, t}^{\mathrm{RS}} \cdot\left(r_{g, t}^{\mathrm{UP}}+r_{g, t}^{\mathrm{DN}}\right)-\sum_{d=1}^{N_{d}} U_{d, t}^{\mathrm{RS}} \cdot\left(r_{d, t}^{\mathrm{UP}}+r_{d, t}^{\mathrm{DN}}\right) \\
& +X_{t}^{\mathrm{DA}} \forall t
\end{aligned}
$$

On the other hand, from d.1)- d.2 and d.5)-

$$
\begin{aligned}
& \lambda_{t}^{\mathrm{EN}}=\widehat{o}_{s, t}^{\mathrm{dis}}+\bar{\mu}_{s, t}^{\mathrm{dis}}-\underline{\mu}_{s, t}^{\mathrm{dis}}-\mu_{s, t}^{\mathrm{dis}, \mathrm{DN}} \quad \forall s, \forall t \\
& \lambda_{t}^{\mathrm{EN}}=\widehat{o}_{s, t}^{\mathrm{ch}}-\bar{\mu}_{s, t}^{\mathrm{ch}}+\underline{\mu}_{s, t}^{\mathrm{ch}}+\mu_{s, t}^{\mathrm{ch}, \mathrm{UP}} \quad \forall s, \forall t \\
& \lambda_{t}^{\mathrm{UP}}=\widehat{o}_{s, t}^{\mathrm{dis}, \mathrm{UP}}+\bar{\mu}_{s, t}^{\mathrm{dis}, \mathrm{UP}}-\underline{\mu}_{s, t}^{\mathrm{dis}, \mathrm{UP}} \quad \forall s, \forall t \\
& \lambda_{t}^{\mathrm{DN}}=\widehat{o}_{s, t}^{\mathrm{dis}, \mathrm{DN}}+\bar{\mu}_{s, t}^{\mathrm{dis}, \mathrm{DN}}-\underline{\mu}_{s, t}^{\mathrm{dis}, \mathrm{DN}}+\mu_{s, t}^{\mathrm{dis}, \mathrm{DN}} \\
& \forall s, \forall t \\
& \lambda_{t}^{\mathrm{UP}}=\widehat{o}_{s, t}^{\mathrm{ch}, \mathrm{UP}}+\bar{\mu}_{s, t}^{\mathrm{ch}, \mathrm{UP}}-\underline{\mu}_{s, t}^{\mathrm{ch}, \mathrm{UP}}+\mu_{s, t}^{\mathrm{ch}, \mathrm{UP}} \\
& \forall s, \forall t \\
& \lambda_{t}^{\mathrm{DN}}=\widehat{o}_{s, t}^{\mathrm{ch}, \mathrm{DN}}+\bar{\mu}_{s, t}^{\mathrm{ch}, \mathrm{DN}}-\underline{\mu}_{s, t}^{\mathrm{ch}, \mathrm{DN}} \quad \forall s, \forall t
\end{aligned}
$$

thus,

$$
\begin{aligned}
& \lambda_{t}^{\mathrm{EN}} \cdot p_{s, t}^{\mathrm{dis}}=\widehat{o}_{s, t}^{\mathrm{dis}} \cdot p_{s, t}^{\mathrm{dis}}+\bar{\mu}_{s, t}^{\text {dis }} \cdot p_{s, t}^{\mathrm{dis}}-\underline{\mu}_{s, t}^{\mathrm{dis}} \cdot p_{s, t}^{\mathrm{dis}} \\
& -\mu_{s, t}^{\mathrm{dis}, \mathrm{DN}} \cdot p_{s, t}^{\mathrm{dis}} \forall s, \forall t \\
& \lambda_{t}^{\mathrm{EN}} \cdot p_{s, t}^{\mathrm{ch}}=\widehat{o}_{s, t}^{\mathrm{ch}} \cdot p_{s, t}^{\mathrm{ch}}-\bar{\mu}_{s, t}^{c h} \cdot p_{s, t}^{\mathrm{ch}}+\underline{\mu}_{s, t}^{\mathrm{ch}} \cdot p_{s, t}^{\mathrm{ch}} \\
& +\mu_{s, t}^{\mathrm{ch}, \mathrm{UP}} \cdot p_{s, t}^{\mathrm{ch}} \forall s, \forall t \\
& \lambda_{t}^{\mathrm{UP}} \cdot r_{s, t}^{\mathrm{dis}, \mathrm{UP}}=\widehat{o}_{s, t}^{\mathrm{dis}, \mathrm{UP}} \cdot r_{s, t}^{\mathrm{dis}, \mathrm{UP}}+\bar{\mu}_{s, t}^{\mathrm{dis}, \mathrm{UP}} \cdot r_{s, t}^{\mathrm{dis}, \mathrm{UP}} \\
& -\underline{\mu}_{s, t}^{\mathrm{dis}, \mathrm{UP}} \cdot r_{s, t}^{\mathrm{dis}, \mathrm{UP}} \quad \forall s, \forall t \\
& \lambda_{t}^{\mathrm{DN}} \cdot r_{s, t}^{\mathrm{dis}, \mathrm{DN}}=\widehat{o}_{s, t}^{\mathrm{dis}, \mathrm{DN}} \cdot r_{s, t}^{\mathrm{dis}, \mathrm{DN}}+\bar{\mu}_{s, t}^{\mathrm{dis}, \mathrm{DN}} \cdot r_{s, t}^{\mathrm{dis}, \mathrm{DN}} \\
& -\mu_{s, t}^{\mathrm{dis}, \mathrm{DN}} \cdot r_{s, t}^{\mathrm{dis}, \mathrm{DN}}+\mu_{s, t}^{\mathrm{dis}, \mathrm{DN}} \cdot r_{s, t}^{\mathrm{dis}, \mathrm{DN}} \forall s, \forall t \\
& \lambda_{t}^{\mathrm{UP}} \cdot r_{s, t}^{\mathrm{ch}, \mathrm{UP}}=\widehat{o}_{s, t}^{\mathrm{ch}, \mathrm{UP}} \cdot r_{s, t}^{\mathrm{ch}, \mathrm{UP}}+\bar{\mu}_{s, t}^{\mathrm{ch}, \mathrm{UP}} \cdot r_{s, t}^{\mathrm{ch}, \mathrm{UP}} \\
& -\underline{\mu}_{s, t}^{c h, U P} \cdot r_{s, t}^{\mathrm{ch}, \mathrm{UP}}+\mu_{s, t}^{\mathrm{ch}, \mathrm{UP}} \cdot r_{s, t}^{\mathrm{ch}, \mathrm{UP}} \forall s, \forall t \\
& \lambda_{t}^{\mathrm{DN}} \cdot r_{s, t}^{\mathrm{ch}, \mathrm{DN}}=\widehat{o}_{s, t}^{\mathrm{ch}, \mathrm{DN}} \cdot r_{s, t}^{\mathrm{ch}, \mathrm{DN}}+\bar{\mu}_{s, t}^{\mathrm{ch}, \mathrm{DN}} \cdot r_{s, t}^{\mathrm{ch}, \mathrm{DN}} \\
& -\underline{\mu}_{s, t}^{\mathrm{ch}, \mathrm{DN}} \cdot r_{s, t}^{\mathrm{ch}, \mathrm{DN}} \forall s, \forall t
\end{aligned}
$$

Additionally, from d.17, d.19, d.21, d.23, d.25, d.27, d.29, and d.30:

$$
\begin{aligned}
& \underline{\mu}_{s, t}^{\mathrm{dis}} \cdot p_{s, t}^{\mathrm{dis}}=0 ; \quad \underline{\mu}_{s, t}^{\mathrm{ch}} \cdot p_{s, t}^{\mathrm{ch}}=0 \quad \forall s, \forall t \\
& \underline{\mu}_{s, t}^{\mathrm{dis}, \mathrm{UP}} \cdot r_{s, t}^{\mathrm{dis}, \mathrm{UP}}=0 ; \quad \underline{\mu}_{s, t}^{\mathrm{dis}, \mathrm{DN}} \cdot r_{s, t}^{\mathrm{dis}, \mathrm{DN}}=0 \quad \forall s, \forall t \\
& \underline{\mu}_{s, t}^{\mathrm{ch}, \mathrm{UP}} \cdot r_{s, t}^{\mathrm{ch}, \mathrm{UP}}=0 ; \quad \underline{\mu}_{s, t}^{\mathrm{ch}, \mathrm{DN}} \cdot r_{s, t}^{\mathrm{ch}, \mathrm{DN}}=0 \quad \forall s, \forall t \\
& \underline{\mu}_{s, t}^{\mathrm{dis}} \cdot \cdot_{s, t}^{\mathrm{dis}}=r_{s, t}^{\mathrm{dis}, \mathrm{DN}} ; \quad \underline{\mu}_{s, t}^{c h} \cdot p_{s, t}^{\mathrm{ch}}=r_{s, t}^{\mathrm{ch}, \mathrm{UP}} \quad \forall s, \forall t
\end{aligned}
$$


Using (f.16)-(f.25) to simplify (f.9) yields

$$
\begin{aligned}
& -\sum_{s=1}^{N_{s}}\left(\lambda_{t}^{\mathrm{EN}} \cdot p_{s, t}^{\mathrm{ch}}-\lambda_{t}^{\mathrm{EN}} \cdot p_{s, t}^{\mathrm{dis}}-\lambda_{t}^{\mathrm{UP}} \cdot r_{s, t}^{\mathrm{ch}, \mathrm{UP}}\right. \\
& \left.-\lambda_{t}^{\mathrm{DN}} \cdot r_{s, t}^{\mathrm{ch}, \mathrm{DN}}-\lambda_{t}^{U P} \cdot r_{s, t}^{\mathrm{dis}, \mathrm{UP}}-\lambda_{t}^{\mathrm{DN}} \cdot r_{s, t}^{\mathrm{dis}, \mathrm{DN}}\right) \\
& =-\sum_{d=1}^{N_{d}} U_{d, t}^{\mathrm{EN}} \cdot p_{d, t}+\sum_{g=1}^{N_{g}} O_{g, t}^{\mathrm{EN}} \cdot p_{g, t} \\
& +\sum_{g=1}^{N_{g}} O_{g, t}^{\mathrm{RS}} \cdot\left(r_{g, t}^{\mathrm{UP}}+r_{g, t}^{\mathrm{DN}}\right)+\sum_{d=1}^{N_{d}} U_{d, t}^{\mathrm{RS}} \cdot\left(r_{d, t}^{\mathrm{UP}}+r_{d, t}^{\mathrm{DN}}\right) \\
& +X_{t}^{\mathrm{DA}} \forall t
\end{aligned}
$$

Note that expression f.26 provides a linear equivalent for the summation of all those bilinear terms in the upperlevel objective function (a.1) that include day-ahead variables (i.e., day-ahead prices times day-ahead quantity schedules). Likewise, an equivalent for bilinear terms in a.1) including balancing variables can be obtained. To this end, we formulate the strong duality equation (g.1) below corresponding to each lower-level problem (c):

$$
\begin{aligned}
& \sum_{d=1}^{N_{d}} V_{d, t} \cdot l_{d, t, k}+\sum_{g=1}^{N_{g}} O_{g, t}^{\mathrm{EN}} \cdot\left(q_{g, t, k}^{\mathrm{UP}}-q_{g, t, k}^{\mathrm{DN}}\right) \\
& +\sum_{s=1}^{N_{s}} \widehat{o}_{s, t}^{\mathrm{dis}} \cdot\left(q_{s, t, k}^{\mathrm{dis}, \mathrm{UP}}-q_{s, t, k}^{\mathrm{dis}, \mathrm{DN}}\right)+\sum_{s=1}^{N_{s}} \widehat{o}_{s, t}^{\mathrm{ch}} \cdot\left(q_{s, t, k}^{\mathrm{ch}, \mathrm{UP}}-q_{s, t, k}^{\mathrm{ch}, \mathrm{DN}}\right) \\
& +\sum_{d=1}^{N_{d}} U_{d, t}^{\mathrm{EN}} \cdot\left(q_{d, t, k}^{\mathrm{UP}}-q_{d, t, k}^{\mathrm{DN}}\right)= \\
& -\sum_{s=1}^{N_{s}}\left(\bar{\rho}_{s, t, k}^{\mathrm{dis}, \mathrm{UP}} \cdot r_{s, t}^{\mathrm{dis}, \mathrm{UP}}+\bar{\rho}_{s, t, k}^{\mathrm{dis}, \mathrm{DN}} \cdot r_{s, t}^{\mathrm{dis}, \mathrm{DN}}\right. \\
& \left.+\bar{\rho}_{s, t, k}^{\mathrm{ch}, \mathrm{UP}} \cdot r_{s, t}^{\mathrm{ch}, \mathrm{UP}}+\bar{\rho}_{s, t, k}^{\mathrm{ch}, \mathrm{DN}} \cdot r_{s, t}^{\mathrm{ch}, \mathrm{DN}}\right)+X_{t, k}^{\mathrm{BL}} \forall t, \forall k \quad(\mathrm{~g} \cdot 1) \\
& \quad \text { where, } \\
& X_{t, k}^{\mathrm{BL}}=-\sum_{g=1}^{N_{g}}\left(\bar{\rho}_{g, t, k}^{\mathrm{RU}} \cdot r_{g, t}^{\mathrm{UP}}+\bar{\rho}_{g, t}^{\mathrm{DN}} \cdot r_{g, t}^{\mathrm{DN}}\right)-\sum_{d=1}^{N_{d}}\left(\bar{\rho}_{d, t, k} \cdot p_{d, t}\right. \\
& \left.+\bar{\rho}_{d, t, k}^{\mathrm{UP}} \cdot r_{d, t}^{\mathrm{UP}}+\bar{\rho}_{d, t, k}^{\mathrm{DN}} \cdot r_{d, t}^{\mathrm{DN}}\right)+\lambda_{t, k}^{\mathrm{BL}} \cdot Q_{t, k}^{\mathrm{BL}} \forall t, \forall k \quad(\mathrm{~g} .2)
\end{aligned}
$$

From e.19), e.21), e.23), and e.25):

$$
\begin{aligned}
& \bar{\rho}_{s, t, k}^{\mathrm{dis}, \mathrm{UP}} \cdot r_{s, t}^{\mathrm{dis}, \mathrm{UP}}=\bar{\rho}_{s, t, k}^{\mathrm{dis}, \mathrm{UP}} \cdot q_{s, t, k}^{\mathrm{dis}, \mathrm{UP}} \quad \forall s, \forall t, \forall k \\
& \bar{\rho}_{s, t, k}^{\mathrm{dis}, \mathrm{DN}} \cdot r_{s, t}^{\mathrm{dis} \mathrm{DN}}=\bar{\rho}_{s, t, k}^{\mathrm{dis}, \mathrm{DN}} \cdot q_{s, t, k}^{\mathrm{dis}, \mathrm{DN}} \quad \forall s, \forall t, \forall k \\
& \bar{\rho}_{s, t, k}^{\mathrm{ch}, \mathrm{UP}} \cdot r_{s, t}^{\mathrm{ch}, \mathrm{UP}}=\bar{\rho}_{s, t, k}^{\mathrm{ch}, \mathrm{UP}} \cdot q_{s, t, k}^{\mathrm{ch}, \mathrm{UP}} \quad \forall s, \forall t, \forall k \\
& \bar{\rho}_{s, t, k}^{\mathrm{ch}, \mathrm{DN}} \cdot r_{s, t}^{\mathrm{ch}, \mathrm{DN}}=\bar{\rho}_{s, t, k}^{\mathrm{ch}, \mathrm{DN}} \cdot q_{s, t, k}^{\mathrm{ch}, \mathrm{DN}} \quad \forall s, \forall t, \forall k
\end{aligned}
$$

Substituting g.3 - g.6 in g.1 results in

$$
\begin{aligned}
& \sum_{s=1}^{N_{s}}\left(\left[\widehat{\sigma}_{s, t}^{\mathrm{dis}}+\bar{\rho}_{s, t, k}^{\mathrm{dis}, \mathrm{UP}}\right] \cdot q_{s, t, k}^{\mathrm{dis}, \mathrm{UP}}-\left[\widehat{o}_{s, t}^{\mathrm{dis}}-\bar{\rho}_{s, t, k}^{\mathrm{dis}, \mathrm{DN}}\right] \cdot q_{s, t, k}^{\mathrm{dis}, \mathrm{DN}}\right. \\
& \left.+\left[\widehat{o}_{s, t}^{\mathrm{ch}}+\bar{\rho}_{s, t, k}^{\mathrm{ch}, \mathrm{UP}}\right] \cdot q_{s, t, k}^{\mathrm{ch}, \mathrm{UP}}-\left[\widehat{o}_{s, t}^{\mathrm{ch}}-\bar{\rho}_{s, t, k}^{\mathrm{ch}, \mathrm{DN}}\right] \cdot q_{s, t, k}^{\mathrm{ch}, \mathrm{DN}}\right)=
\end{aligned}
$$

$$
\begin{aligned}
& -\sum_{d=1}^{N_{d}} V_{d, t} \cdot l_{d, t, k}-\sum_{g=1}^{N_{g}} O_{g, t}^{\mathrm{EN}} \cdot\left(q_{g, t, k}^{\mathrm{UP}}-q_{g, t, k}^{\mathrm{DN}}\right) \\
& -\sum_{d=1}^{N_{d}} U_{d, t}^{\mathrm{EN}} \cdot\left(q_{d, t, k}^{\mathrm{UP}}-q_{d, t, k}^{\mathrm{DN}}\right)+X_{t, k}^{\mathrm{BL}} \quad \forall t, \forall k
\end{aligned}
$$

On the other hand, from (e.1)-(e.4):

$$
\begin{aligned}
& \lambda_{t, k}^{\mathrm{BL}}=\widehat{o}_{s, t}^{\mathrm{dis}}+\bar{\rho}_{s, t, k}^{\mathrm{dis}, \mathrm{UP}}-\underline{\rho}_{s, t, k}^{\mathrm{dis}, \mathrm{UP}} \quad \forall s, \forall t, \forall k \\
& \lambda_{t, k}^{\mathrm{BL}}=\widehat{o}_{s, t}^{\mathrm{dis}}-\bar{\rho}_{s, t, k}^{\mathrm{dis}, \mathrm{DN}}+\underline{\rho}_{s, t, k}^{\mathrm{dis}, \mathrm{DN}} \quad \forall s, \forall t, \forall k \\
& \lambda_{t, k}^{\mathrm{BL}}=\widehat{o}_{s, t}^{\mathrm{ch}}+\bar{\rho}_{s, t, k}^{\mathrm{ch}, \mathrm{UP}}-\underline{\rho}_{s, t, k}^{\mathrm{ch}, \mathrm{UP}} \quad \forall s, \forall t, \forall k \\
& \lambda_{t, k}^{\mathrm{BL}}=\widehat{o}_{s, t}^{\mathrm{ch}}-\bar{\rho}_{s, t, k}^{\mathrm{ch}, \mathrm{DN}}+\underline{\rho}_{s, t, k}^{\mathrm{ch} \mathrm{DN}} \quad \forall s, \forall t, \forall k
\end{aligned}
$$

therefore,

$$
\begin{aligned}
& \lambda_{t, k}^{\mathrm{BL}} \cdot q_{s, t, k}^{\mathrm{dis}, \mathrm{UP}}=\widehat{o}_{s, t}^{\mathrm{dis}} \cdot q_{s, t, k}^{\mathrm{dis}, \mathrm{UP}}+\bar{\rho}_{s, t, k}^{\mathrm{dis}, \mathrm{UP}} \cdot q_{s, t, k}^{\mathrm{dis}, \mathrm{UP}} \\
& -\underline{\rho}_{s, t, k}^{\mathrm{dis}, \mathrm{UP}} \cdot q_{s, t, k}^{\mathrm{dis}, \mathrm{UP}} \quad \forall s, \forall t, \forall k \\
& \lambda_{t, k}^{\mathrm{BL}} \cdot q_{s, t, k}^{\mathrm{dis}, \mathrm{DN}}=\widehat{o}_{s, t}^{\mathrm{dis}} \cdot q_{s, t, k}^{\mathrm{dis}, \mathrm{DN}}-\bar{\rho}_{s, t, k}^{\mathrm{dis}, \mathrm{DN}} \cdot q_{s, t, k}^{\mathrm{dis}, \mathrm{DN}} \\
& +\underline{\rho}_{s, t, k}^{\mathrm{dis}, \mathrm{DN}} \cdot q_{s, t, k}^{\mathrm{dis}, \mathrm{DN}} \quad \forall s, \forall t, \forall k \\
& \lambda_{t, k}^{\mathrm{BL}} \cdot q_{s, t, k}^{\mathrm{ch}, \mathrm{UP}}=\widehat{o}_{s, t}^{\mathrm{ch}} \cdot q_{s, t, k}^{\mathrm{ch}, \mathrm{UP}}+\bar{\rho}_{s, t, k}^{\mathrm{ch}, \mathrm{UP}} \cdot q_{s, t, k}^{\mathrm{ch}, \mathrm{UP}} \\
& -\underline{\rho}_{s, t, k}^{\mathrm{ch}, \mathrm{UP}} \cdot q_{s, t, k}^{\mathrm{ch}, \mathrm{UP}} \quad \forall s, \forall t, \forall k \\
& \lambda_{t, k}^{\mathrm{BL}} \cdot q_{s, t, k}^{\mathrm{ch}, \mathrm{DN}}=\widehat{o}_{s, t}^{\mathrm{ch}} \cdot q_{s, t, k}^{\mathrm{ch}, \mathrm{DN}}-\bar{\rho}_{s, t, k}^{\mathrm{ch}, \mathrm{DN}} \cdot q_{s, t, k}^{\mathrm{ch}, \mathrm{DN}} \\
& +\underline{\rho}_{s, t, k}^{\mathrm{ch}, \mathrm{DN}} \cdot q_{s, t, k}^{\mathrm{ch}, \mathrm{DN}} \forall s, \forall t, \forall k
\end{aligned}
$$

Additionally, from (e.18), e.20), e.22), and e.24):

$$
\begin{array}{ll}
\underline{\rho}_{s, t, k}^{\mathrm{dis}, \mathrm{UP}} \cdot q_{s, t, k}^{\mathrm{dis}, \mathrm{UP}}=0 & \forall s, \forall t, \forall k \\
\underline{\rho}_{s, t, k}^{\mathrm{dis}, \mathrm{DN}} \cdot q_{s, t, k}^{\mathrm{dis}, \mathrm{DN}}=0 & \forall s, \forall t, \forall k \\
\underline{\rho}_{s, t, k}^{\mathrm{ch}, \mathrm{UP}} \cdot q_{s, t, k}^{\mathrm{ch}, \mathrm{UP}}=0 & \forall s, \forall t, \forall k \\
\underline{\rho}_{s, t, k}^{\mathrm{ch}, \mathrm{DN}} \cdot q_{s, t, k}^{\mathrm{ch}, \mathrm{DN}}=0 & \forall s, \forall t, \forall k
\end{array}
$$

Using (g.12)-g.19) to simplify (g.7) concludes

$$
\begin{aligned}
& \sum_{s=1}^{N_{s}}\left(\lambda_{t, k}^{\mathrm{BL}} \cdot q_{s, t, k}^{\mathrm{dis}, \mathrm{UP}}-\lambda_{t, k}^{\mathrm{BL}} \cdot q_{s, t, k}^{\mathrm{dis}, \mathrm{DN}}\right. \\
& \left.+\lambda_{t, k}^{\mathrm{BL}} \cdot q_{s, t, k}^{\mathrm{ch}, \mathrm{UP}}-\lambda_{t, k}^{\mathrm{BL}} \cdot q_{s, t, k}^{\mathrm{ch}, \mathrm{DN}}\right)= \\
& -\sum_{d=1}^{N_{d}} V_{d, t} \cdot l_{d, t, k}-\sum_{g=1}^{N_{g}} O_{g, t}^{\mathrm{EN}} \cdot\left(q_{g, t, k}^{\mathrm{UP}}-q_{g, t, k}^{\mathrm{DN}}\right) \\
& -\sum_{d=1}^{N_{d}} U_{d, t}^{\mathrm{EN}} \cdot\left(q_{d, t, k}^{\mathrm{UP}}-q_{d, t, k}^{\mathrm{DN}}\right)+X_{t, k}^{\mathrm{BL}} \quad \forall t, \forall k
\end{aligned}
$$

We now rewrite the upper-level objective function a.1 using (f.26) and (g.20) as follows:

\section{Min.}

$$
\sum_{t=1}^{N_{t}}\left[\sum_{s=1}^{N_{s}}\left(M C_{s}^{\mathrm{ch}} \cdot p_{s, t}^{\mathrm{ch}}+M C_{s}^{\mathrm{dis}} \cdot p_{s, t}^{\mathrm{dis}}\right)\right.
$$




$$
\begin{aligned}
& -\sum_{d=1}^{N_{d}} U_{d, t}^{\mathrm{EN}} \cdot p_{d, t}+\sum_{g=1}^{N_{g}} O_{g, t}^{\mathrm{EN}} \cdot p_{g, t} \\
& +\sum_{g=1}^{N_{g}} O_{g, t}^{\mathrm{RS}} \cdot\left(r_{g, t}^{\mathrm{UP}}+r_{g, t}^{\mathrm{DN}}\right)+\sum_{d=1}^{N_{d}} U_{d, t}^{\mathrm{RS}} \cdot\left(r_{d, t}^{\mathrm{UP}}+r_{d, t}^{\mathrm{DN}}\right)-X_{t}^{\mathrm{DA}} \\
& +\sum_{k=1}^{N_{k}} \Phi_{k} \cdot\left[-M C_{s}^{\mathrm{ch}} \cdot\left(q_{s, t, k}^{\mathrm{ch}, \mathrm{UP}}-q_{s, t, k}^{\mathrm{ch}, \mathrm{DN}}\right)\right. \\
& +M_{s}^{\mathrm{dis}} \cdot\left(q_{s, t, k}^{\mathrm{dis}, \mathrm{UP}}-q_{s, t, k}^{\mathrm{dis}, \mathrm{DN}}\right) \\
& +\sum_{d=1}^{N_{d}} V_{d, t} \cdot l_{d, t, k}+\sum_{g=1}^{N_{g}} O_{g, t}^{\mathrm{EN}} \cdot\left(q_{g, t, k}^{\mathrm{UP}}-q_{g, t, k}^{\mathrm{DN}}\right) \\
& \left.\left.+\sum_{d=1}^{N_{d}} U_{d, t} \cdot\left(q_{d, t, k}^{\mathrm{UP}}-q_{d, t, k}^{\mathrm{DN}}\right)-X_{t, k}^{\mathrm{BL}}\right]\right]
\end{aligned}
$$

Note that there are still a few bilinear terms in (h.1) within $X_{t, k}^{\mathrm{BL}}$; each one includes a day-ahead quantity schedule and a balancing dual variable. These bilinear terms are $\bar{\rho}_{g, t, k}^{\mathrm{UP}} \cdot r_{g, t}^{\mathrm{UP}}$, $\bar{\rho}_{g, t, k}^{\mathrm{DN}} \cdot r_{g, t}^{\mathrm{DN}}, \bar{\rho}_{d, t, k}^{\mathrm{UP}} \cdot r_{d, t}^{\mathrm{UP}}, \bar{\rho}_{d, t, k}^{\mathrm{DN}} \cdot r_{d, t}^{\mathrm{DN}}$ and $\rho_{d, t, k} \cdot p_{d, t}$. We use a binary expansion method [20] to linearize each of those bilinear terms but at the cost of introducing an approximation. For instance, the steps for linearizing the bilinear term $\bar{\rho}_{g, t, k}^{\mathrm{UP}} \cdot r_{g, t}^{\mathrm{UP}}$ are as follows:

1) The primal variable $r_{g, t}^{\mathrm{UP}}$ is selected to be discretized since its lower and upper bounds are known. We discretize $r_{g, t}^{\mathrm{UP}}$ by parameters $\widetilde{r}_{g, t, i}^{\mathrm{UP}}$, where $i$ is an index for discrete values.

2) The bilinear term $\bar{\rho}_{g, t, k}^{\mathrm{UP}} \cdot r_{g, t}^{\mathrm{UP}}$ is replaced by $\sum_{i} \bar{\eta}_{g, t, k, i}^{\mathrm{UP}} \cdot \widetilde{r}_{g, t, i}^{\mathrm{UP}}, \quad$ where $\bar{\eta}_{g, t, k, i}^{\mathrm{UP}}$ is an auxiliary continuous variable.

3) Among the discrete values $\widetilde{r}_{g, t, i}^{\mathrm{UP}}$, the following equation selects the closest one to the original variable $r_{g, t}^{\mathrm{UP}}$ :

$$
\begin{aligned}
r_{g, t}^{\mathrm{UP}}-\frac{\Delta r_{g, t}}{2} \leq \sum_{i} \bar{u}_{g, t, k, i}^{\mathrm{UP}} \widetilde{r}_{g, t, i}^{\mathrm{UP}} \leq r_{g, t}^{\mathrm{UP}}+\frac{\Delta r_{g, t}}{2} \\
\forall g, \forall t, \forall k
\end{aligned}
$$

where constant $\Delta r_{g, t}$ is the MW-step, i.e., it shows the distance of two sequential discrete values. In addition, $\bar{u}_{g, t, k, i}^{\mathrm{UP}}$ is an auxiliary binary variable, which determines the closest discrete value to the original variable $r_{g, t}^{\mathrm{UP}}$. Note that $\sum_{i} \bar{u}_{g, t, k, i}^{\mathrm{UP}}=1 \quad \forall g, \forall t, \forall k$.

4) In addition, the following constraints are incorporated into the problem:

$$
\begin{aligned}
& 0 \leq\left[\bar{\rho}_{g, t, k}^{\mathrm{UP}}-\bar{\eta}_{g, t, k, i}^{\mathrm{UP}}\right] \leq M .\left(1-\bar{u}_{g, t, k, i}^{\mathrm{UP}}\right) \quad \forall g, \forall t, \forall k, \forall i \\
& 0 \leq \bar{\eta}_{g, t, k, i}^{\mathrm{UP}} \leq M \cdot \bar{u}_{g, t, k, i}^{\mathrm{UP}} \quad \forall g, \forall t, \forall k, \forall i
\end{aligned}
$$

where $M$ is a large enough positive value.

\section{REFERENCES}

[1] Federal Energy Regulatory Commission (FERC), "Third-party provision of ancillary services; accounting and financial reporting for new electric storage technologies," Jul. 2013. [Online]. Available: http: //www.ferc.gov/whats-new/comm-meet/2013/071813/E-22.pdf
[2] E. Drury, P. Denholm, and R. Sioshansi, "The value of compressed air energy storage in energy and reserve markets," Energy, vol. 36, no. 8, pp. 4959-4973, Apr. 2011.

[3] J. Eichman, P. Denholm, J. Jorgenson, and U. Helman, "Operational benefits of meeting californias energy storage targets," Technical Results, National Renewable Energy Laboratory (NREL), Dec. 2015.

[4] R. Sioshansi, P. Denholm, T. Jenkin, and J. Weiss, "Estimating the value of electricity storage in PJM: Arbitrage and some welfare effects," Energy Economics, vol. 31, no. 2, pp. 269-277, Mar. 2009.

[5] H. Mohsenian-Rad, "Coordinated price-maker operation of large energy storage units in nodal energy markets," IEEE Transactions on Power Systems, vol. 31, no. 1, pp. 786-797, Jan. 2016.

[6] E. Nasrolahpour, S. J. Kazempour, H. Zareipour, and W. D. Rosehart, "Strategic sizing of energy storage facilities in electricity markets," IEEE Transactions on Sustainable Energy, vol. 7, no. 4, pp. 1462-1472, Oct. 2016.

[7] — "Impacts of ramping inflexibility of conventional generators on strategic operation of energy storage facilities," IEEE Transactions on Smart Grid, to be published, 2016.

[8] G. He, Q. Chen, C. Kang, P. Pinson, and Q. Xia, "Optimal bidding strategy of battery storage in power markets considering performancebased regulation and battery cycle life," IEEE Transactions on Smart Grid, vol. 7, no. 5, pp. 2359-2367, Sep. 2016.

[9] H. Ding, P. Pinson, Z. Hu, and Y. Song, "Integrated bidding and operating strategies for wind-storage systems," IEEE Transactions on Sustainable Energy, vol. 7, no. 1, pp. 163-172, Jan. 2016.

[10] R. Moreno, R. Moreira, and G. Strbac, "A MILP model for optimising multi-service portfolios of distributed energy storage," Applied Energy, vol. 137, pp. $554-566,2015$.

[11] H. Akhavan-Hejazi and H. Mohsenian-Rad, "Optimal operation of independent storage systems in energy and reserve markets with high wind penetration," IEEE Transactions on Smart Grid, vol. 5, no. 2, pp. 1088-1097, Mar. 2014.

[12] X. He, E. Delarue, W. D'haeseleer, and J.-M. Glachant, "A novel business model for aggregating the values of electricity storage," Energy Policy, vol. 39, no. 3, pp. 1575 - 1585, 2011.

[13] A. D. Lamont, "Assessing the economic value and optimal structure of large-scale electricity storage," IEEE Transactions on Power Systems, vol. 28, no. 2, pp. 911-921, May 2013.

[14] Pennsylvania-New Jersey-Maryland Interconnection (PJM), "PJM manual 11: Energy \& ancillary services market operations," Mar. 2016. [Online]. Available: http://www.pjm.com/ /media/documents/manuals/ m11-redline.ashx

[15] —, "Integration of wind production forecasting into day ahead and real-time scheduling and commitment," Apr. 2013. [Online]. Available: https://www.pjm. $\mathrm{com} / \sim /$ media/committees-groups/subcommittees/irs/20130617/ 20130617-item-02-irtf-issue-resolution-wind-forecasting-tool.ashx

[16] D. Pozo, E. Sauma, and J. Contreras, "Basic theoretical foundations and insights on bilevel models and their applications to power systems," Annals of Operations Research, to be published, 2017.

[17] A. Nemirovsk and A. Shapiro, "Convex approximations of chance constrained programs," SIAM Journal on Optimization, vol. 17, no. 4 pp. 969-996, Nov. 2006.

[18] J. Fortuny-Amat and B. McCarl, "A representation and economic interpretation of a two-level programming problem," The Journal of the Operational Research Society, vol. 32, no. 9, pp. 783-792, Sep. 1981.

[19] C. Ruiz and A. J. Conejo, "Pool strategy of a producer with endogenous formation of locational marginal prices," IEEE Transactions on Power Systems, vol. 24, no. 4, pp. 1855-1866, Nov. 2009.

[20] C. Ruiz, A. J. Conejo, and S. A. Gabriel, "Pricing non-convexities in an electricity pool," IEEE Transactions on Power Systems, vol. 27, no. 3, pp. 1334-1342, Aug. 2012.

[21] D. Kirschen and G. Strbac, Fundamentals of Power System Economics. John Wiley \& Sons, Ltd, 2004.

[22] S. Oren, "Auction design for ancillary reserve products," IEEE Power Engineering Society Summer Meeting, 2002.

[23] S. Dempe, Foundations of Bilevel Programming. Kluwer Academic Publishers, 2002.

[24] Alberta Electric System Operator (AESO), "Energy storage integration," 2014. [Online]. Available: http://www.aeso.ca/downloads/ EnergyStorageIntegrationDiscussionPaper.pdf

[25] W. L. Oliveira, C. Sagastizabal, D. J. Penna, M. E. Maceira, and J. M. Damazio, "Optimal scenario tree reduction for stochastic stream flows in power generation planning problems," Optimization Methods and Software, vol. 25, pp. 917-936, 2010. 
[26] S. J. Kazempour, A. J. Conejo, and C. Ruiz, "Strategic generation investment considering futures and spot markets," IEEE Transactions on Power Systems, vol. 27, no. 3, pp. 1467-1476, Aug. 2012.

Ehsan Nasrolahpour (S'14) received the B.Sc. degree from the Shahid Chamran University of Ahvaz, Ahvaz, Iran, in 2010, the M.Sc. degree from University of Tehran, Tehran, Iran, in 2013. He is currently pursuing his Ph.D. at University of Calgary, Calgary, AB, Canada. His research interests include power systems, electricity markets, and operations research.

Jalal Kazempour (M'14) is an Assistant Professor in the Department of Electrical Engineering, Technical University of Denmark, Kgs. Lyngby, Denmark. $\mathrm{He}$ received his Ph.D. degree in Electrical Engineering from University of Castilla-La Mancha, Ciudad Real, Spain, in 2013. He was a postdoctoral fellow at The Johns Hopkins University, MD, USA, in 2014, and at the Technical University of Denmark in 2015-2016. His research interests include power systems, electricity markets, optimization and its applications to energy systems.

Hamidreza Zareipour (S'03-M'07-SM'09) received the Ph.D. degree in electrical engineering from the University of Waterloo, Waterloo, ON, Canada, in 2006. He is currently a full Professor with the Department of Electrical and Computer Engineering, University of Calgary, Calgary, AB, Canada. His research focuses on economics, planning, and management of power and energy systems in a deregulated electricity market environment.

William D. Rosehart (SM'05) received the B.Sc, M.Sc., and Ph.D. degrees in electrical engineering from the University of Waterloo, Waterloo, ON, Canada. Currently, he is the Dean of Schulich School of Engineering at the University of Calgary, Calgary, AB, Canada. His main research interests are in the areas of numerical optimization techniques, power system stability, and modeling power systems in a deregulated environment. 